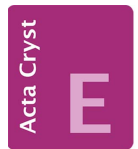

CRYSTALLOGRAPHIC COMMUNICATIONS

ISSN 2056-9890

Received 8 October 2015

Accepted 27 October 2015

Edited by M. Weil, Vienna University of Technology, Austria

Keywords: crystal structure; erbium complexes 4-aminobenzoic acid; 4-chloro-3-nitrobenzoic acid; coordinating dimethyl sulfoxide; hydrogen bonding

CCDC references: 1433543; 1433542 Supporting information: this article has supporting information at journals.iucr.org/e

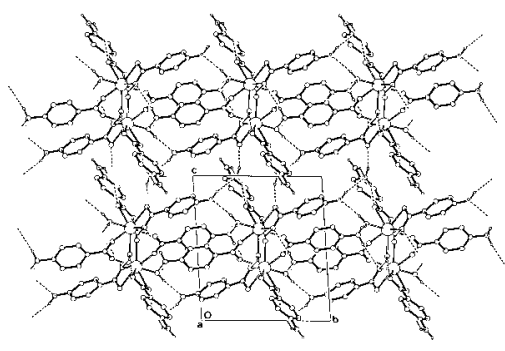

OPEN $\odot$ ACCESS

\section{Crystal structures of two erbium(III) complexes with 4-aminobenzoic acid and 4-chloro-3-nitro- benzoic acid}

\author{
Graham Smith $^{\mathrm{a} *}$ and Daniel E. Lynch ${ }^{\mathrm{b}}$
}

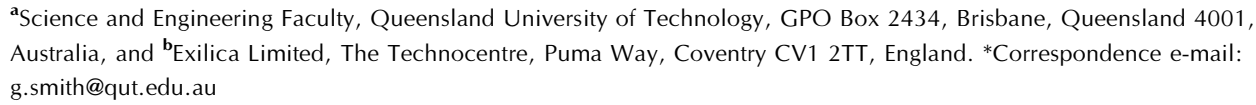

The crystal structures of two erbium(III) complexes with 4-aminobenzoic acid (4-ABAH), namely bis $\left(\mu_{2}\right.$-4-aminobenzoato- $\left.\kappa^{2} O: O^{\prime}\right)$ bis[bis(4-aminobenzoato$\left.\kappa^{2} O, O^{\prime}\right)$ diaquaerbium(III)] dihydrate, $\left[\mathrm{Er}_{2}\left(\mathrm{C}_{7} \mathrm{H}_{6} \mathrm{NO}_{2}\right)_{6}\left(\mathrm{H}_{2} \mathrm{O}\right)_{4}\right] \cdot 2 \mathrm{H}_{2} \mathrm{O}$, (I), and 4-chloro-3-nitrobenzoic acid (CLNBAH), namely poly[hexakis $\left(\mu_{2}\right.$-4-chloro-3nitrobenzoato- $\left.\kappa^{2} O: O^{\prime}\right)$ bis(dimethyl sulfoxide- $\left.\kappa O\right)$ dierbium(III)], $\left[\mathrm{Er}_{2}\left(\mathrm{C}_{7} \mathrm{H}_{3} \mathrm{Cl}\right.\right.$ $\left.\left.\mathrm{NO}_{4}\right)_{6}\left(\mathrm{C}_{2} \mathrm{H}_{6} \mathrm{OS}\right)_{2}\right]_{n}$, (II), have been determined. In the structure of solvatomorphic compound (I), the symmetry-related irregular $\mathrm{ErO}_{8}$ coordination polyhedra in the discrete centrosymmetric dinuclear complex comprise two monodentate water molecules and six carboxylate O-atom donors, four from two bidentate carboxylate $O, O^{\prime}$-chelate groups and two from the bismonodentate $O: O^{\prime}$-bridging group of the third 4-ABA anion. The $\mathrm{Er}-\mathrm{O}$ bond-length range is $2.232(3)-2.478$ (3) $\AA$ and the Er $\cdots$ Er separation in the dinuclear complex unit is 4.7527 (4) $\AA$. One of the coordinating water molecules is involved in an intra-unit $\mathrm{O}-\mathrm{H} \cdots \mathrm{O}$ hydrogen-bonding association with an inversion-related carboxylate O-atom acceptor. In contrast, the anhydrous compound (II) is polymeric, based on centrosymmetric dinuclear repeat units comprising $\mathrm{ErO}_{7}$ coordination polyhedra which involve four O-atom donors from two bidentate $O: O^{\prime}$-bridging carboxylate groups, one $\mathrm{O}$-atom donor from the monodentate dimethyl sulfoxide ligand and two O-atom donors from the third bridging CLNBA anion. The latter provides the inter-unit link in the onedimensional coordination polymer extending along [100]. The $\mathrm{Er}-\mathrm{O}$ bondlength range in (II) is $2.239(6)-2.348$ (6) $\AA$ and the Er...Er separation within the dinuclear unit is 4.4620 (6) $\AA$. In the crystal of (I), extensive inter-dimer $\mathrm{O}-$ $\mathrm{H} \cdots \mathrm{O}$ and $\mathrm{N}-\mathrm{H} \cdots \mathrm{O}$ hydrogen-bonding interactions involving both the coordinating water molecules and the solvent water molecules, as well as the amine groups of the 4-ABA anions, give an overall three-dimensional network structure. Within this structure are also weak $\pi-\pi$ ring interactions between two of the coordinating ligands [ring-centroid separations $=3.676(3)$ and $3.711(2) \AA]$. With (II), only weak intra-polymer $\mathrm{C}-\mathrm{H} \cdots \mathrm{O}, \mathrm{C}-\mathrm{H} \cdots \mathrm{Cl}$ and $\mathrm{C}-\mathrm{H} \cdots \mathrm{S}$ interactions are present.

\section{Chemical context}

The coordination chemistry of the rare earth $(R E)$ metals has been investigated extensively and the structures of a large number of complexes with various ligand types are known (Sastri et al., 2003). Of interest is the lanthanide contraction across the series and 4-aminobenzoic acid (4-ABAH) has provided a valuable ligand for this purpose in a comprehensive study of this effect with the $R E^{3+}(\mathrm{La}-\mathrm{Y})$ series of complexes (Sun et al., 2004). Within this series there are two sub-sets of isotypic complexes, one monoclinic $\left(P 2_{1} / n\right)(\mathrm{La}-\mathrm{Tb}$ as well as Dy and Er), in which the structures are twodimensional, the second triclinic $(P \overline{1})$ forming dinuclear structures ( $\mathrm{Yb}, \mathrm{Lu}, \mathrm{Y}$, as well as $\mathrm{Tb})$. The solvatomorphism of 
the $\mathrm{Tb}$ member (monoclinic, $\left[\mathrm{Tb}_{2}(4-\mathrm{ABA})_{6}\left(\mathrm{H}_{2} \mathrm{O}\right)_{2}\right]$; triclinic $\left.\left[\left[\mathrm{Tb}_{2}(4-\mathrm{ABA})_{6}\left(\mathrm{H}_{2} \mathrm{O}\right)_{2}\right] \cdot 2 \mathrm{H}_{2} \mathrm{O}\right]\right\}$ is of interest and its occurrence was indicated as being dependent on $\mathrm{pH}$ control in the preparation.
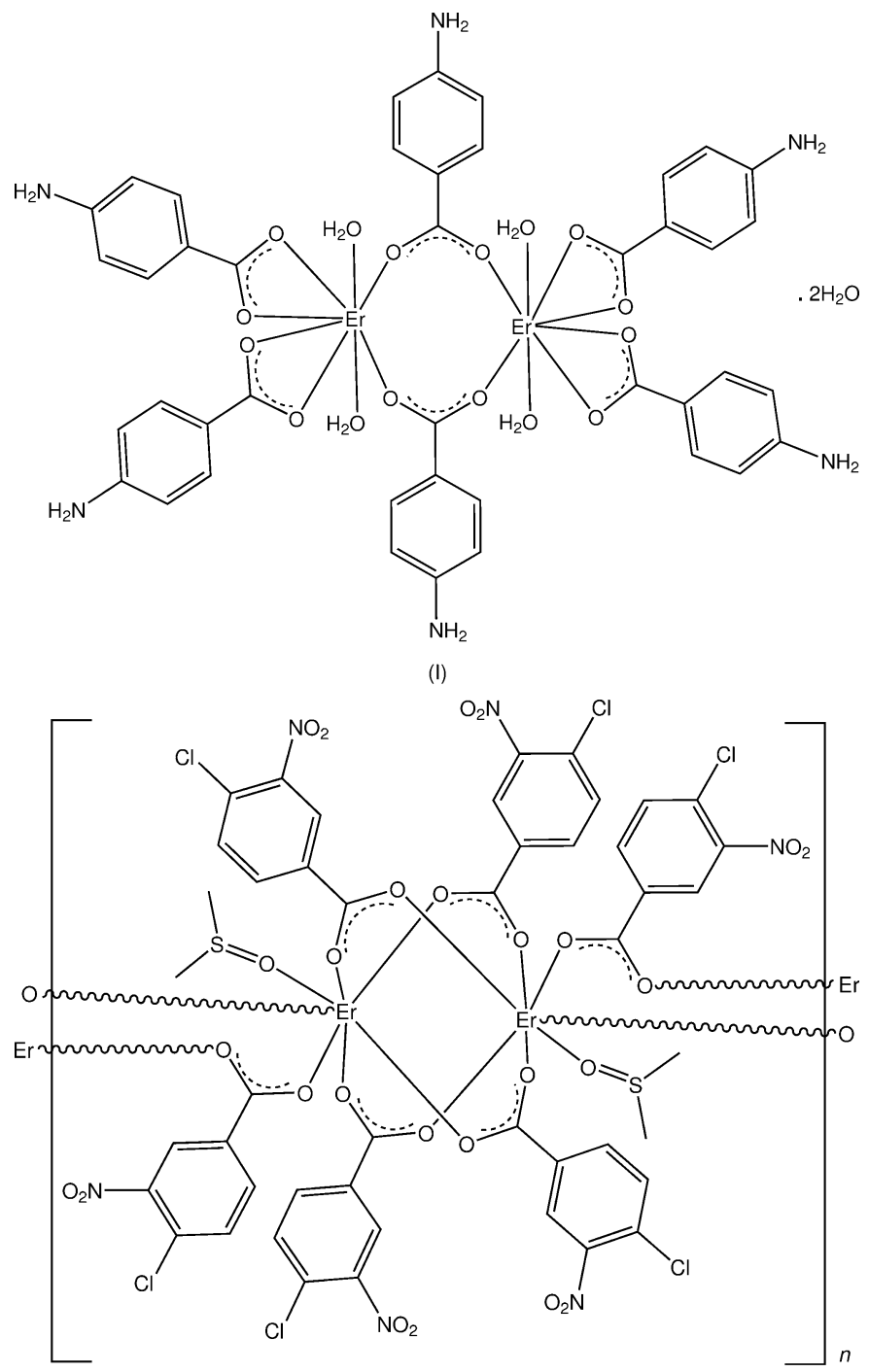

(II)

It was considered that some of the other later members of the $R E$ series (predominantly triclinic) might also show the same effect so this was tested with $\mathrm{Er}$ in a reaction of erbium(III) acetate with 4-ABA in aqueous ethanol under mild reaction conditions, with no additional $\mathrm{pH}$ control. The title triclinic complex $\left[\mathrm{Er}_{2}\left(\mathrm{C}_{7} \mathrm{H}_{6} \mathrm{NO}_{2}\right)_{6}\left(\mathrm{H}_{2} \mathrm{O}\right)_{4}\right] \cdot 2 \mathrm{H}_{2} \mathrm{O}$, (I), was obtained. For (I), the preliminary unit-cell data (Table 1) suggested a possible solvatomorphic variant of the previously reported polymeric monoclinic $\mathrm{Er}^{3+}$ complex with 4-ABA (Sun et al., 2004), and this was confirmed in the X-ray structural analysis. The comparative cell data for the triclinic $\mathrm{Tb}^{3+}$ complex with 4-ABA are $a=9.0964$ (1), $b=11.0117$ (1), $c=$ 12.7430 (2) $\AA, \alpha=89.372$ (5), $\beta=72.0360$ (6), $\gamma=75.0730(7)^{\circ}$, $V=1169.97$ (2) $\AA^{3}$, confirming that the two are isotypic.

Complex (II), anhydrous $\left[\mathrm{Er}_{2}\left(\mathrm{C}_{7} \mathrm{H}_{3} \mathrm{ClNO}_{4}\right)_{6}\left(\mathrm{C}_{2} \mathrm{H}_{6} \mathrm{OS}\right)_{2}\right]_{n}$, was obtained in a similar reaction to (I), using erbium(III) acetate and 4-chloro-3-nitrobenzoic acid (CLNBAH), with
Table 1

Selected bond lengths $(\AA)$ for $(\mathrm{I})$.

\begin{tabular}{llll}
\hline Er1-O1W & $2.373(2)$ & Er1-O12A & $2.333(3)$ \\
Er1-O2W & $2.295(3)$ & Er1-O12B & $2.385(3)$ \\
Er1-O11A & $2.477(3)$ & Er1-O12C & $2.232(3)$ \\
Er1-O11B & $2.478(3)$ & Er1-O11C & $2.233(4)$ \\
\hline
\end{tabular}

Symmetry code: (i) $-x+1,-y+1,-z+1$.

subsequent recrystallization using DMSO. The structures of both complexes are reported herein.

\section{Structural commentary}

In the title centrosymmetric dinuclear structure of compound (I) (Fig. 1), the two identical irregular $\mathrm{ErO}_{8}$ complex units [Er-O bond length range, 2.232 (3)-2.478 (3) ^] (Table 1), comprise two monodentate water molecules $(\mathrm{O} 1 W, \mathrm{O} 2 W)$, four $\mathrm{O}$-atom donors from two slightly asymmetric bidentate $O, O$ ' chelate carboxylate groups (the $A$ and $B$ 4-ABA ligands) and two bridging $\mathrm{O}$-atom donors from two symmetry-related ligands $(C)$. The Er $\cdots \operatorname{Er}^{\mathrm{i}}$ separation in the dinuclear unit is 4.7527 (4) $\AA$. Unlike the polymeric solvatomorphic $\mathrm{Er}^{\mathrm{III}}$ complex $\left[\mathrm{Er}_{2}(4-\mathrm{ABA})_{6}\left(\mathrm{H}_{2} \mathrm{O}\right)_{2}\right]_{n} \cdot n \mathrm{H}_{2} \mathrm{O}$ (Sun et al., 2004), in which the extending $\mathrm{Er}-\mathrm{N}$ bond is somewhat elongated at 2.660 (3) $\AA$, with (I), there is no reasonable $\mathrm{Er}-\mathrm{N}$ bonding contact. The monodentate water molecule $\mathrm{O} 2 \mathrm{~W}$ in (I) replaces the bridging amino $\mathrm{N}$-donor site which is present in the 8-coordination sphere about $\mathrm{Er}$ in the solvatopolymorph. Within the dinuclear complex unit of (I), an intra-dimer $\mathrm{O}-$ $\mathrm{H} \cdot \mathrm{O}_{\text {carboxylate }}$ hydrogen bond is present between one of the the coordinating water molecules $(\mathrm{O} 1 W)$ and an inversion-

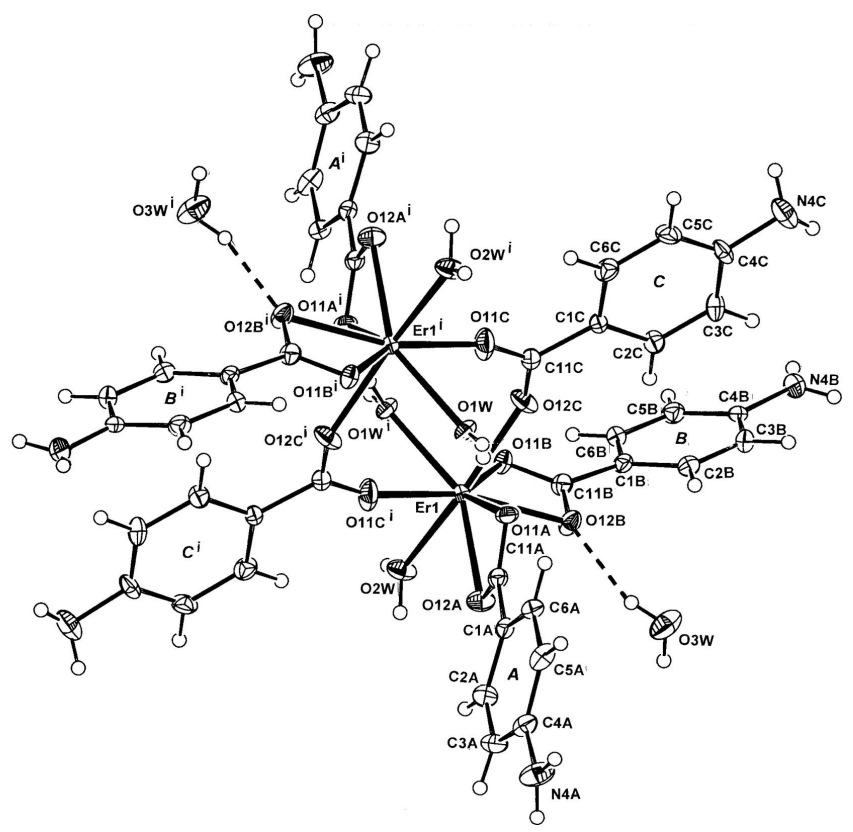

Figure 1

The molecular configuration and atom-naming scheme for the centrosymmetric dinuclear title complex and water molecules of solvation in (I), with displacement ellipsoids drawn at the $40 \%$ probability level. For symmetry code (i), see Table 1 . 
Table 2

Hydrogen-bond geometry $\left(\AA{ }^{\circ}{ }^{\circ}\right)$ for (I).

\begin{tabular}{lclll}
\hline$D-\mathrm{H} \cdots A$ & $D-\mathrm{H}$ & $\mathrm{H} \cdots A$ & $D \cdots A$ & $D-\mathrm{H} \cdots A$ \\
\hline $\mathrm{O} 1 W-\mathrm{H} 11 W \cdots \mathrm{O} 11 A^{\mathrm{i}}$ & $0.82(4)$ & $1.95(4)$ & $2.757(4)$ & $166(4)$ \\
$\mathrm{O} 1 W-\mathrm{H} 12 W \cdots \mathrm{O} 11 B^{\text {ii }}$ & $0.82(3)$ & $1.98(3)$ & $2.777(4)$ & $163(4)$ \\
$\mathrm{O} 2 W-\mathrm{H} 21 W \cdots \mathrm{N} 4 B^{\text {iii }}$ & $0.84(4)$ & $2.09(4)$ & $2.902(5)$ & $162(5)$ \\
$\mathrm{O} 2 W-\mathrm{H} 22 W \cdots \mathrm{N} 4 C^{\text {iv }}$ & $0.86(4)$ & $1.89(4)$ & $2.735(6)$ & $168(5)$ \\
$\mathrm{O} 3 W-\mathrm{H} 31 W \cdots \mathrm{O} 12 B$ & $0.83(4)$ & $1.99(4)$ & $2.777(4)$ & $160(5)$ \\
$\mathrm{O} 3 W-\mathrm{H} 32 W \cdots \mathrm{O} 12 A^{\mathrm{v}}$ & $0.85(5)$ & $2.07(5)$ & $2.841(5)$ & $151(5)$ \\
$\mathrm{N} 4 A-\mathrm{H} 42 A \cdots \mathrm{O} 3 W^{\text {vi }}$ & $0.88(4)$ & $2.08(4)$ & $2.902(6)$ & $156(4)$ \\
$\mathrm{N} 4 B-\mathrm{H} 41 B \cdots \mathrm{O} 3 W^{\text {vii }}$ & $0.86(4)$ & $2.18(4)$ & $3.014(6)$ & $164(4)$ \\
$\mathrm{N} 4 C-\mathrm{H} 42 C \cdots \mathrm{O} 11 B^{\text {viii }}$ & $0.86(3)$ & $2.49(4)$ & $3.341(5)$ & $170(5)$ \\
\hline
\end{tabular}

Symmetry codes: (i) $-x+1,-y+1,-z+1$; (ii) $-x+2,-y+1,-z+1$; (iii) $x, y-1, z$; (iv) $x+1, y-1, z$; (v) $-x+1,-y+1,-z+2$; (vi) $-x,-y+1,-z+2$; (vii) $-x+1,-y+2,-z+2$; (viii) $-x+1,-y+2,-z+1$.

related carboxylate $\mathrm{O}$-atom $\left(\mathrm{O} 11 A^{\mathrm{i}}\right)$ (Table 2). This structure is similar to the triclinic isotypic $\mathrm{Tb}^{3+}$ complex with 4-ABA (Sun et al., 2004).

In (I), the 4-ABA ligand species show some variation in the conformation of the carboxylate groups. In one of the bidentate $O, O^{\prime}$-chelate ligands $(A)$ and the bridging ligand $(C)$, the groups are essentially coplanar with the benzene ring [torsion angles $\mathrm{C} 2 A / C-\mathrm{C} 1 A / C-\mathrm{C} 11 A / C-\mathrm{O} 11 A / C=$ $171.2(4)$ and $174.8(4)^{\circ}$, respectively], while in the second bidentate chelate ligand $(B)$ the group is rotated out of the plane [corresponding torsion angle $=155.9(4)^{\circ}$ ]. Such a 'planar' conformation is also found in the structure of the parent acid (Gracin \& Fischer, 2005) and in molecular adducts with aromatic carboxylic acids (Chadwick et al., 2009).

In the crystal structure of complex (II), a centrosymmetric dinuclear repeat unit is present with the two inversion-related $\mathrm{Er}^{\mathrm{III}}$ atoms (Fig. 2) being seven-coordinated through four bridging carboxylate $O, O^{1}$ groups (the $A$ and $B$ ligands), a

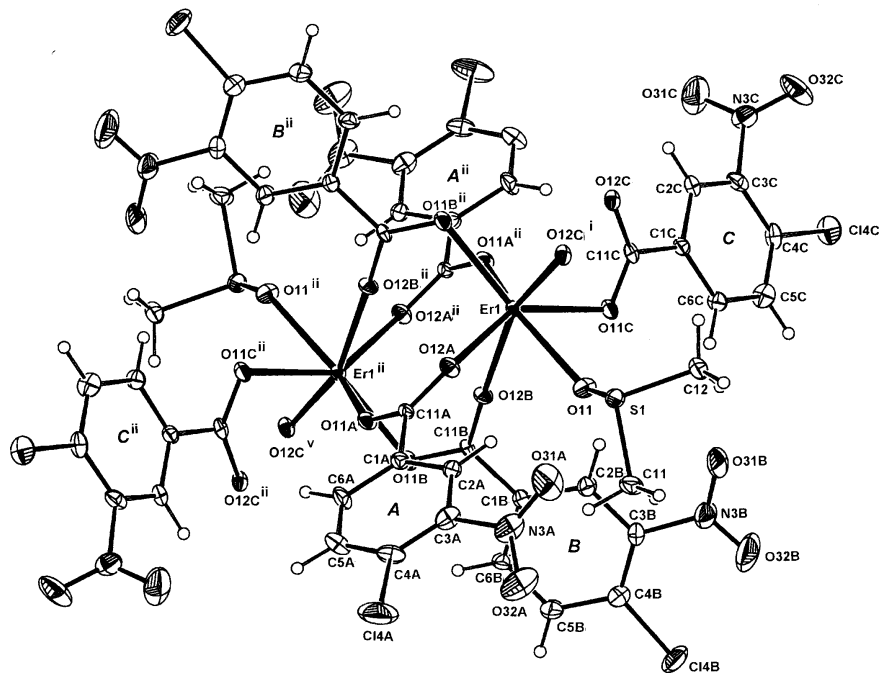

Figure 2

The molecular configuration and atom-naming scheme for the centrosymmetric dinuclear repeat unit in the polymeric complex (II), with displacement ellipsoids drawn at the $40 \%$ probability level. [Symmetry code: (v) $x+1, y, z$; for other symmetry codes, see Table 3.]
Table 3

Selected bond lengths ( $\mathrm{A})$ for (II).

\begin{tabular}{llll}
\hline Er1-O11 & $2.306(7)$ & Er1-O12 $C^{\mathrm{i}}$ & $2.287(6)$ \\
Er1-O11C & $2.312(8)$ & Er1-O11A & $2.300(6)$ \\
Er1-O12A & $2.317(7)$ & Er1-O11B & $2.348(6)$ \\
Er1-O12B & $2.239(6)$ & & \\
\hline
\end{tabular}

Symmetry codes: (i) $-x,-y+1,-z+1$; (ii) $-x+1,-y+1,-z+1$.

Table 4

Hydrogen-bond geometry $\left(\AA,^{\circ}\right)$ for (II).

\begin{tabular}{lllll}
\hline$D-\mathrm{H} \cdots A$ & $D-\mathrm{H}$ & $\mathrm{H} \cdots A$ & $D \cdots A$ & $D-\mathrm{H} \cdots A$ \\
\hline $\mathrm{C} 2 A-\mathrm{H} 2 A \cdots \mathrm{S} 1$ & 0.95 & 2.86 & $3.743(10)$ & 155 \\
$\mathrm{C} 2 B-\mathrm{H} 2 B \cdots \mathrm{O} 11$ & 0.95 & 2.56 & $3.298(13)$ & 135 \\
$\mathrm{C} 11-\mathrm{H} 111 \cdots \mathrm{C} 14 A^{\mathrm{iii}}$ & 0.98 & 2.79 & $3.486(11)$ & 129 \\
$\mathrm{C} 12-\mathrm{H} 123 \cdots \mathrm{O} 32 A^{\text {iv }}$ & 0.98 & 2.44 & $3.376(15)$ & 158 \\
\hline
\end{tabular}

Symmetry codes: (iii) $-x+1,-y+1,-z$; (iv) $-x,-y+1,-z$.

monodentate DMSO O-atom and O-donors $\left(\mathrm{O} 12 \mathrm{C}^{\mathrm{i}}\right)$ and $\mathrm{O} 11 C^{\mathrm{i}}$ from the $C$ ligand which extends the dinuclear unit into a one-dimensional coordination polymer lying along [100] (Fig. 3). The Er-O bond length range is 2.239 (6)-2.348 (6) (Table 3) and the Er..E Er $^{\text {ii }}$ separation within the dimeric unit is 4.4620 (6) $\AA$. Also present within the repeat unit are a $\mathrm{C} 2 B-\mathrm{H} \cdots \mathrm{O} 11$ hydrogen bond [3.298 (13) $\AA$ ] ] and a C2AH...S1 interaction [3.743 (10) A] (Table 4).

The torsion angles defining the conformation of the carboxylate groups of the CLNBA ligands in (II) are $\mathrm{C} 2 A / B$ / $C-\mathrm{C} 1 A / B / C-\mathrm{C} 11 A / B / C-\mathrm{O} 11 A / B / C=158.7$ (9), $177.2(9)$ and $160.3(8)^{\circ}$, respectively. The torsion angles of the nitro groups $\mathrm{C} 2 A / B / C-\mathrm{C} 3 A / B / C-\mathrm{N} 3 A / B / C-\mathrm{O} 32 A / B / C$ are $-150.4(12), 174.1(16)$ and $120.3(13)^{\circ}$, respectively. In the structure of the parent CLNBAH acid (Ishida \& Fukunaga, 2003), the corresponding torsion angles are 174.02 (17) and $-132.61(18)^{\circ}$ compared to 179.7 (2) and $-137.8(2)^{\circ}$ in the Na-CLNBA monohydrate salt (Smith, 2013).

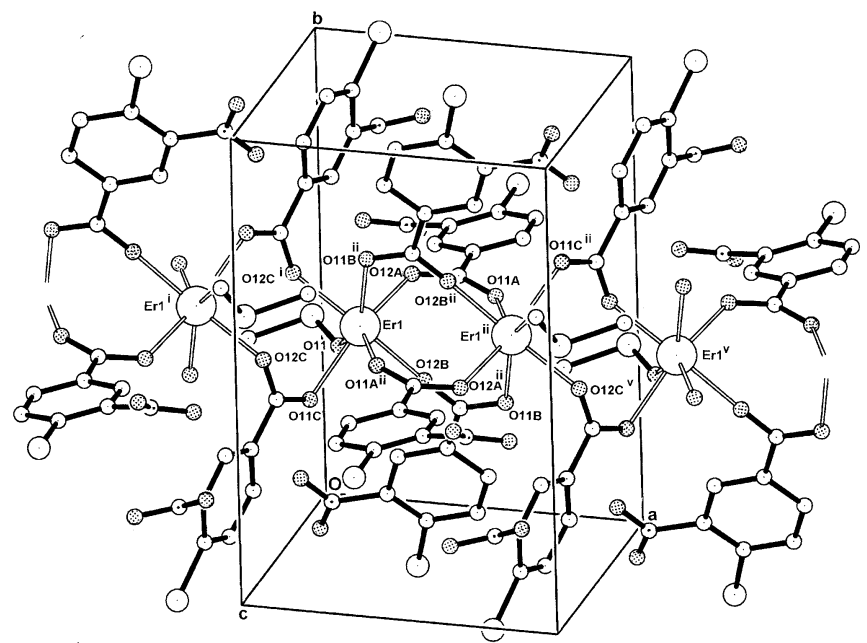

Figure 3

The packing of the one-dimensional polymeric chain structure of (II) in the unit cell, viewed approximately along [001]. $\mathrm{H}$ atoms have been omitted. 


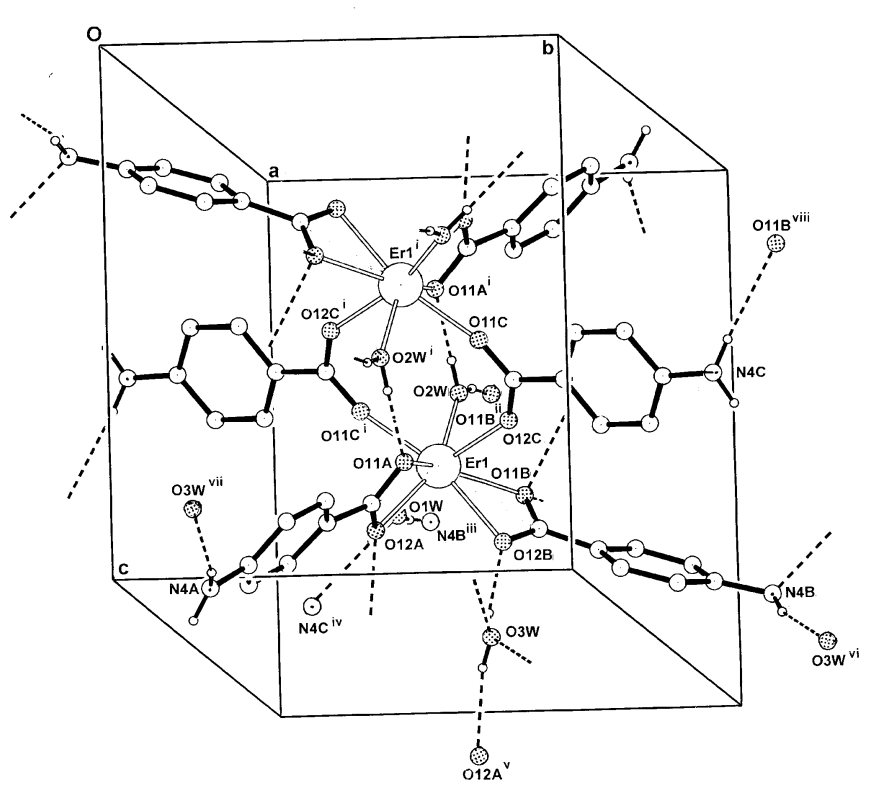

Figure 4

The dimeric complex (I) in the unit cell, viewed approximately down [100], showing intra- and interdimer hydrogen-bonding extensions as dashed lines. Non-associative $\mathrm{H}$ atoms have been omitted. For symmetry codes, see Table 2.

\section{Supramolecular features}

In the crystal structure of compound (I), extensive inter-unit $\mathrm{O}-\mathrm{H} \cdots \mathrm{O}$ and $\mathrm{O}-\mathrm{H} \cdots \mathrm{N}$ hydrogen-bonding interactions are present, involving both the coordinating water molecules as well as the solvent water molecules, with carboxylate $\mathrm{O}$-atom acceptors and amine $\mathrm{N}$-atom acceptors (Table 2). These, together with amine $\mathrm{N}-\mathrm{H} \cdots \mathrm{O}_{\text {water }}$ and $\mathrm{O}_{\text {carboxyl }}$ hydrogen bonds give a three-dimensional network structure (Figs. 4 and $5)$. One $\mathrm{H}$ atom of each of the amine groups on the three 4ABA ligand components of the complex is not involved in hydrogen-bonding. Also present in the supramolecular structure are weak $\pi-\pi$ interactions between $A$ ligands [ringcentroid separation $\left.A \cdots A^{\mathrm{vii}}=3.711(3) \AA\right]$ and $C$ ligands $\left[C \cdots C^{\text {viii }}=3.676(3) \AA\right]$ (for symmetry codes, see Table 2). This dimeric carboxylate-bridged complex mode is similar to that found in the erbium acetate complex $\left[\mathrm{Er}_{2}\left(\mathrm{CH}_{3} \mathrm{CO}_{2}\right)_{6^{-}}\right.$ $\left.\left(\mathrm{H}_{2} \mathrm{O}\right)_{4}\right]_{2} \cdot 6 \mathrm{H}_{2} \mathrm{O}$ (Sawase et al., 1984).

With (II), present are two weak intra-polymer $\mathrm{C}-\mathrm{H} \cdots \mathrm{O}$ hydrogen bonds involving methyl $\mathrm{H}$ atoms and both a DMSO $\mathrm{O}$-atom acceptor and a Cl-atom acceptor (Table 4).

\section{Synthesis and crystallization}

The title compounds were synthesized by warming together for $10 \mathrm{~min}$, a solution obtained by mixing $5 \mathrm{ml}$ of ethanolic 4-aminobenzoic acid (1 mmol: $135 \mathrm{mg}$ ) [for (I)] or 4-chloro-3nitrobenzoic acid (1 mmol: $200 \mathrm{mg}$ ) [for (II)], with $10 \mathrm{ml}$ of aqueous erbium(III) acetate hexahydrate $(0.3 \mathrm{mmol}: 216 \mathrm{mg})$. Partial room-temperature evaporation of these solutions provided pale-pink block-like single crystals of (I), suitable for $\mathrm{X}$-ray analysis while a colourless powder was obtained from

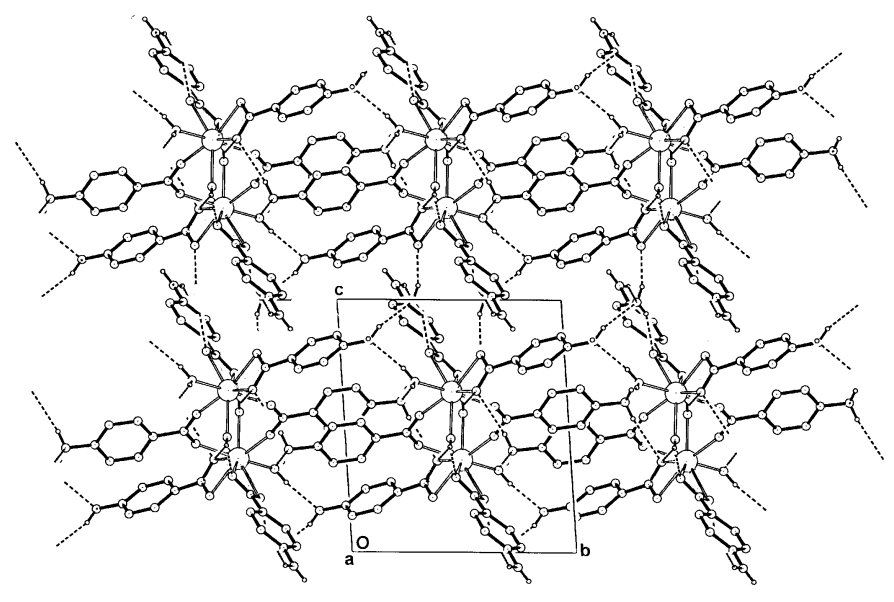

Figure 5

The three-dimensional hydrogen-bonded structure of (I) in the unit cell, viewed along [100]. Non-associative $\mathrm{H}$ atoms have been omitted.

the preparation of (II). Recrystallization using the slow diffusion of water into a DMSO solution gave minor small crystals of (II), suitable for X-ray analysis.

\section{Refinement details}

Crystal data, data collection and structure refinements for (I) and (II) are summarized in Table 5. Hydrogen atoms on all water molecules and the amine groups of the 4-ABA ligands in (I) were located by difference methods and positional parameters were refined with restraints $[\mathrm{O}-\mathrm{H}$ bond length = 0.85 (2) $\AA$ and $\mathrm{N}-\mathrm{H}=0.88$ (2) $\AA$ ] , with $U_{\text {iso }}(\mathrm{H})=1.5 U_{\text {eq }}(\mathrm{O})$ or $1.2 U_{\text {eq }}(\mathrm{N})$. Other $\mathrm{H}$ atoms were included in the refinement at calculated positions $[\mathrm{C}-\mathrm{H}($ aromatic $)=0.95 \AA$ or $\mathrm{C}-$ $\mathrm{H}($ methyl $)=0.96 \AA$, with $U_{\text {iso }}(\mathrm{H})=1.2 U_{\text {eq }}(\mathrm{C})$ (aromatic) or $1.5 U_{\text {eq }}(\mathrm{C})($ methyl $\left.)\right]$, using a riding-model approximation. In the refinement of (II), a number of large difference electron density residual peaks $\left(5-7\right.$ e $\left.\AA^{-3}\right)$ located within $1.0 \AA$ of the Er1 site were present. These are possibly due to poor crystal quality coupled to effects of an insufficient absorption correction.

\section{Acknowledgements}

The author acknowledges support from the Science and Engineering Faculty, Queensland University of Technology.

\section{References}

Agilent (2013). CrysAlis PRO. Agilent Technologies Ltd, Yarnton, England.

Altomare, A., Cascarano, G., Giacovazzo, C. \& Guagliardi, A. (1993). J. Appl. Cryst. 26, 343-350.

Chadwick, K., Sadiq, G., Davey, R. J., Seaton, C. C., Pritchard, R. G. \& Parkin, A. (2009). Cryst. Growth Des. 9, 1278-1279.

Farrugia, L. J. (2012). J. Appl. Cryst. 45, 849-854.

Gracin, S. \& Fischer, A. (2005). Acta Cryst. E61, o1242-o1244.

Ishida, H. \& Fukunaga, T. (2003). Acta Cryst. E59, o1984-o1986.

Sastri, V. S., Bünzli, J.-C., Ramachandra Rao, V., Rayudi, G. V. S. \& Perumareddi, J. R. (2003). In Modern Aspects of Rare Earths and Their Complexes. Amsterdam: Elsevier. 
Table 5

Experimental details.

(I)

\section{Crystal data}

Chemical formula

$M_{\mathrm{r}}$

Crystal system, space group

Temperature (K)

$a, b, c(\AA)$

$\alpha, \beta, \gamma\left({ }^{\circ}\right)$

$V\left(\AA^{3}\right)$

$Z$

Radiation type

$\mu\left(\mathrm{mm}^{-1}\right)$

Crystal size (mm)

Data collection

Diffractometer

Absorption correction

$T_{\min }, T_{\max }$

No. of measured, independent and observed

$[I>2 \sigma(I)]$ reflections

$R_{\text {int }}$

$(\sin \theta / \lambda)_{\max }\left(\AA^{-1}\right)$

Refinement

$R\left[F^{2}>2 \sigma\left(F^{2}\right)\right], w R\left(F^{2}\right), S$

No. of reflections

No. of parameters

No. of restraints

$\mathrm{H}$-atom treatment

$\Delta \rho_{\max }, \Delta \rho_{\min }\left(\mathrm{e} \AA^{-3}\right)$

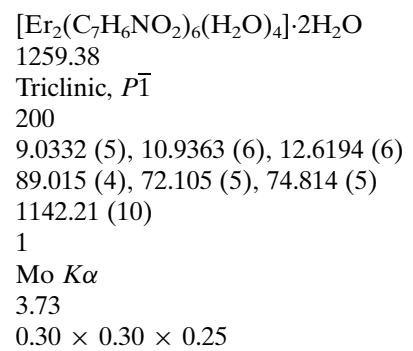

Oxford Diffraction Gemini-S CCD detector

Multi-scan (CrysAlis PRO; Agilent, 2013)

$0.713,0.980$

$7274,4480,4137$

0.035

0.617

$0.029,0.058,1.05$

4480

343

12

$\mathrm{H}$ atoms treated by a mixture of independent and constrained refinement

$1.03,-0.71$
(II)

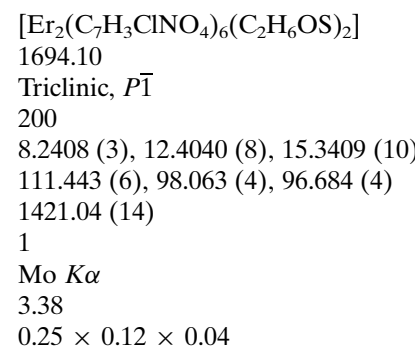

Oxford Diffraction Gemini-S CCD detector Multi-scan (CrysAlis PRO; Agilent, 2013) $0.494,0.980$

$10041,5566,4814$

0.055

0.617

$0.067,0.181,1.06$

5566

397

0

H-atom parameters constrained

$6.83,-2.41$

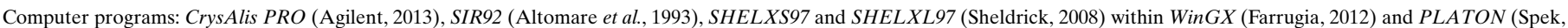
2009).

Sawase, H., Koizumi, Y., Suzuki, Y., Shimoi, M. \& Ouchi, Z. (1984).

Bull. Chem. Soc. Jpn, 57, 2730-2737.

Sheldrick, G. M. (2008). Acta Cryst. A64, 112-122.

Smith, G. (2013). Acta Cryst. C69, 1472-1477.
Spek, A. L. (2009). Acta Cryst. D65, 148-155.

Sun, H.-L., Ye, C.-H., Wang, X.-Y., Li, J.-R., Gao, S. \& Yu, K.-B. (2004). J. Mol. Struct. 702, 77-83. 


\section{supporting information}

Acta Cryst. (2015). E71, 1457-1461［https://doi.org/10.1107/S2056989015020319]

\section{Crystal structures of two erbium(III) complexes with 4-aminobenzoic acid and 4-chloro-3-nitrobenzoic acid}

\section{Graham Smith and Daniel E. Lynch}

Computing details

For both compounds, data collection: CrysAlis PRO (Agilent, 2013); cell refinement: CrysAlis PRO (Agilent, 2013); data reduction: CrysAlis PRO (Agilent, 2013). Program(s) used to solve structure: SIR92 (Altomare et al., 1993) for (I); SHELXS97 (Sheldrick, 2008) for (II). For both compounds, program(s) used to refine structure: SHELXL97 (Sheldrick, 2008) within $\operatorname{Win} G X$ (Farrugia, 2012); molecular graphics: PLATON (Spek, 2009); software used to prepare material for publication: PLATON (Spek, 2009).

(I) $\operatorname{Bis}\left(\mu_{2}-4\right.$-aminobenzoato- $\left.\kappa^{2} O: O^{\prime}\right)$ bis [bis $\left(4\right.$-aminobenzoato- $\left.\kappa^{2} O, O^{\prime}\right)$ diaquaerbium(III)] dihydrate

Crystal data

$\left[\mathrm{Er}_{2}\left(\mathrm{C}_{7} \mathrm{H}_{6} \mathrm{NO}_{2}\right)_{6}\left(\mathrm{H}_{2} \mathrm{O}\right)_{4}\right] \cdot 2 \mathrm{H}_{2} \mathrm{O}$

$M_{r}=1259.38$

Triclinic, $P \overline{1}$

Hall symbol: -P 1

$a=9.0332(5) \AA$

$b=10.9363(6) \AA$

$c=12.6194(6) \AA$

$\alpha=89.015(4)^{\circ}$

$\beta=72.105(5)^{\circ}$

$\gamma=74.814(5)^{\circ}$

$V=1142.21(10) \AA^{3}$

Data collection

Oxford Diffraction Gemini-S CCD-detector diffractometer

Radiation source: Enhance (Mo) X-ray source

Graphite monochromator

Detector resolution: 16.077 pixels $\mathrm{mm}^{-1}$

$\omega$ scans

Absorption correction: multi-scan (CrysAlis PRO; Agilent, 2013)

$T_{\min }=0.713, T_{\max }=0.980$

Refinement

Refinement on $F^{2}$

Least-squares matrix: full

$R\left[F^{2}>2 \sigma\left(F^{2}\right)\right]=0.029$

$w R\left(F^{2}\right)=0.058$

$S=1.05$

4480 reflections
$Z=1$

$F(000)=622$

$D_{\mathrm{x}}=1.831 \mathrm{Mg} \mathrm{m}^{-3}$

Mo $K \alpha$ radiation, $\lambda=0.71073 \AA$

Cell parameters from 3598 reflections

$\theta=3.6-28.8^{\circ}$

$\mu=3.73 \mathrm{~mm}^{-1}$

$T=200 \mathrm{~K}$

Block, pink

$0.30 \times 0.30 \times 0.25 \mathrm{~mm}$

7274 measured reflections

4480 independent reflections

4137 reflections with $I>2 \sigma(I)$

$R_{\text {int }}=0.035$

$\theta_{\text {max }}=26.0^{\circ}, \theta_{\min }=3.4^{\circ}$

$h=-11 \rightarrow 11$

$k=-10 \rightarrow 13$

$l=-15 \rightarrow 14$

343 parameters

12 restraints

Primary atom site location: structure-invariant direct methods

Secondary atom site location: difference Fourier map 
Hydrogen site location: inferred from neighbouring sites

$\mathrm{H}$ atoms treated by a mixture of independent and constrained refinement

$$
\begin{aligned}
& w=1 /\left[\sigma^{2}\left(F_{\mathrm{o}}{ }^{2}\right)+(0.011 P)^{2}\right] \\
& \text { where } P=\left(F_{\mathrm{o}}^{2}+2 F_{\mathrm{c}}{ }^{2}\right) / 3 \\
& (\Delta / \sigma)_{\max }=0.002 \\
& \Delta \rho_{\max }=1.03 \mathrm{e} \AA^{-3} \\
& \Delta \rho_{\min }=-0.71 \mathrm{e} \AA^{-3}
\end{aligned}
$$

Special details

Geometry. Bond distances, angles etc. have been calculated using the rounded fractional coordinates. All su's are estimated from the variances of the (full) variance-covariance matrix. The cell e.s.d.'s are taken into account in the estimation of distances, angles and torsion angles

Refinement. Refinement of $F^{2}$ against ALL reflections. The weighted $R$-factor $w R$ and goodness of fit $S$ are based on $F^{2}$,

\begin{tabular}{|c|c|c|c|c|}
\hline & $x$ & $y$ & $z$ & $U_{\text {iso }} * / U_{\text {eq }}$ \\
\hline Er1 & $0.63868(2)$ & $0.48651(2)$ & $0.63400(1)$ & $0.0172(1)$ \\
\hline $\mathrm{O} 1 \mathrm{~W}$ & $0.8216(3)$ & $0.4689(3)$ & $0.4504(2)$ & $0.0236(9)$ \\
\hline $\mathrm{O} 2 \mathrm{~W}$ & $0.8310(4)$ & $0.3257(3)$ & 0.6738 & $0.0310(10)$ \\
\hline O3W & $0.4420(4)$ & $0.6614(4)$ & $1.0062(3)$ & $0.0450(13)$ \\
\hline O11A & $0.3424(3)$ & $0.5182(3)$ & $0.7029(2)$ & $0.0229(9)$ \\
\hline O11B & $0.8438(3)$ & $0.5973(3)$ & $0.6381(2)$ & $0.0226(9)$ \\
\hline $\mathrm{O} 11 \mathrm{C}$ & $0.4044(4)$ & $0.6642(3)$ & $0.4607(3)$ & $0.0393(11)$ \\
\hline $\mathrm{O} 12 \mathrm{~A}$ & $0.4967(3)$ & $0.3977(3)$ & $0.7885(2)$ & $0.0279(10)$ \\
\hline O12B & $0.6239(4)$ & 0.6268 & $0.7818(2)$ & $0.0297(10)$ \\
\hline $\mathrm{O} 12 \mathrm{C}$ & $0.5398(3)$ & $0.6760(3)$ & $0.5771(2)$ & $0.0326(10)$ \\
\hline N4A & $-0.1513(5)$ & $0.2669(5)$ & $1.0592(3)$ & $0.0420(16)$ \\
\hline N4B & $0.8254(5)$ & $1.1355(4)$ & $0.8371(3)$ & $0.0338(14)$ \\
\hline $\mathrm{N} 4 \mathrm{C}$ & $0.1613(5)$ & $1.2581(4)$ & $0.5966(4)$ & $0.0408(14)$ \\
\hline $\mathrm{C} 1 \mathrm{~A}$ & $0.2234(5)$ & $0.3912(4)$ & $0.8436(3)$ & $0.0205(12)$ \\
\hline C1B & $0.7719(5)$ & $0.7812(4)$ & 0.7614 & $0.0209(11)$ \\
\hline $\mathrm{C} 1 \mathrm{C}$ & $0.3588(4)$ & $0.8633(4)$ & 0.5499 & $0.0173(11)$ \\
\hline $\mathrm{C} 2 \mathrm{~A}$ & $0.2533(5)$ & $0.2916(4)$ & $0.9109(3)$ & $0.0259(12)$ \\
\hline $\mathrm{C} 2 \mathrm{~B}$ & $0.6425(5)$ & $0.8743(4)$ & $0.8302(3)$ & $0.0245(12)$ \\
\hline $\mathrm{C} 2 \mathrm{C}$ & $0.3840(5)$ & $0.9328(4)$ & 0.6308 & $0.0239(12)$ \\
\hline $\mathrm{C} 3 \mathrm{~A}$ & $0.1314(5)$ & $0.2489(4)$ & 0.9799 & $0.0286(16)$ \\
\hline $\mathrm{C} 3 \mathrm{~B}$ & $0.6601(5)$ & $0.9903(4)$ & $0.8557(3)$ & $0.0269(12)$ \\
\hline $\mathrm{C} 3 \mathrm{C}$ & $0.3173(5)$ & $1.0619(4)$ & $0.6478(3)$ & $0.0297(14)$ \\
\hline $\mathrm{C} 4 \mathrm{~A}$ & $-0.0285(5)$ & $0.3068(4)$ & 0.9855 & $0.0272(16)$ \\
\hline C4B & $0.8090(5)$ & $1.0165(4)$ & 0.8158 & $0.0238(14)$ \\
\hline $\mathrm{C} 4 \mathrm{C}$ & $0.2265(5)$ & $1.1253(4)$ & $0.5836(4)$ & $0.0264(14)$ \\
\hline C5A & $-0.0601(5)$ & $0.4036(4)$ & $0.9147(3)$ & $0.0284(14)$ \\
\hline C5B & $0.9399(5)$ & $0.9232(4)$ & 0.7501 & $0.0263(12)$ \\
\hline $\mathrm{C} 5 \mathrm{C}$ & $0.1958(5)$ & $1.0556(4)$ & 0.5055 & $0.0295(14)$ \\
\hline C6A & $0.0656(5)$ & $0.4452(4)$ & 0.8453 & $0.0240(12)$ \\
\hline C6B & $0.9214(5)$ & $0.8076(4)$ & 0.7221 & $0.0243(12)$ \\
\hline C6C & $0.2620(5)$ & $0.9257(4)$ & 0.4890 & $0.0272(14)$ \\
\hline
\end{tabular}
conventional $R$-factors $R$ are based on $F$, with $F$ set to zero for negative $F^{2}$. The threshold expression of $F^{2}>\sigma\left(F^{2}\right)$ is used only for calculating $R$-factors $(\mathrm{gt})$ etc. and is not relevant to the choice of reflections for refinement. $R$-factors based on $F^{2}$ are statistically about twice as large as those based on $F$, and $R$ - factors based on ALL data will be even larger.

Fractional atomic coordinates and isotropic or equivalent isotropic displacement parameters $\left(\AA^{2}\right)$ 


$\begin{array}{lllll}\text { C11A } & 0.3588(5) & 0.4394(4) & 0.7749(3) & 0.0205(12) \\ \text { C11B } & 0.7480(5) & 0.6613(4) & 0.7262(3) & 0.0222(12) \\ \text { C11C } & 0.4396(5) & 0.7246(4) & 0.5278(3) & 0.0209(12) \\ \text { H2A } & 0.35910 & 0.25320 & 0.90870 & 0.0310^{*} \\ \text { H2B } & 0.54240 & 0.85770 & 0.85940 & 0.0290^{*} \\ \text { H2C } & 0.44660 & 0.89160 & 0.67390 & 0.0290^{*} \\ \text { H3A } & 0.15460 & 0.18120 & 1.02310 & 0.0350^{*} \\ \text { H3B } & 0.57120 & 1.05190 & 0.90000 & 0.0320^{*} \\ \text { H3C } & 0.33360 & 1.10700 & 0.70320 & 0.0350^{*} \\ \text { H5A } & -0.16540 & 0.43970 & 0.91440 & 0.0340^{*} \\ \text { H5B } & 1.04110 & 0.93850 & 0.72460 & 0.0310^{*} \\ \text { H5C } & 0.13050 & 1.09660 & 0.46410 & 0.0350^{*} \\ \text { H6A } & 0.04410 & 0.51030 & 0.79920 & 0.0290^{*} \\ \text { H6B } & 1.00980 & 0.74670 & 0.67650 & 0.0290^{*} \\ \text { H6C } & 0.24110 & 0.87990 & 0.43650 & 0.0330^{*} \\ \text { H11W } & 0.788(5) & 0.467(4) & 0.397(3) & 0.0350^{*} \\ \text { H12W } & 0.917(3) & 0.463(4) & 0.417(3) & 0.0350^{*} \\ \text { H21W } & 0.807(6) & 0.278(4) & 0.726(3) & 0.0460^{*} \\ \text { H22W } & 0.934(3) & 0.314(5) & 0.655(4) & 0.0460^{*} \\ \text { H41A } & -0.136(6) & 0.223(4) & 1.115(3) & 0.0500^{*} \\ \text { H41B } & 0.750(4) & 1.182(4) & 0.891(3) & 0.0400^{*} \\ \text { H41C } & 0.198(6) & 1.289(5) & 0.645(3) & 0.0490^{*} \\ \text { H42A } & -0.248(3) & 0.305(4) & 1.056(4) & 0.0500^{*} \\ \text { H42B } & 0.919(3) & 1.130(5) & 0.843(4) & 0.0400^{*} \\ \text { H42C } & 0.171(6) & 1.288(5) & 0.532(2) & 0.0490^{*} \\ \text { H31W } & 0.507(5) & 0.634(5) & 0.944(3) & 0.0680^{*} \\ \text { H32W } & 0.489(6) & 0.624(5) & 1.051(4) & 0.0680^{*}\end{array}$

Atomic displacement parameters $\left(\AA^{2}\right)$

\begin{tabular}{lllllll}
\hline & $U^{11}$ & $U^{22}$ & $U^{33}$ & $U^{12}$ & $U^{13}$ & $U^{23}$ \\
\hline Er1 & $0.0190(1)$ & $0.0146(1)$ & $0.0170(1)$ & $-0.0037(1)$ & $-0.0048(1)$ & $0.0002(1)$ \\
O1W & $0.0247(16)$ & $0.0303(17)$ & $0.0135(14)$ & $-0.0055(14)$ & $-0.0043(12)$ & $-0.0005(13)$ \\
O2W & $0.0219(16)$ & $0.0321(19)$ & $0.0371(19)$ & $-0.0049(15)$ & $-0.0093(15)$ & $0.0164(15)$ \\
O11A & $0.0254(15)$ & $0.0245(16)$ & $0.0175(14)$ & $-0.0052(13)$ & $-0.0063(12)$ & $0.0069(12)$ \\
O11B & $0.0220(15)$ & $0.0253(16)$ & $0.0201(14)$ & $-0.0065(13)$ & $-0.0057(12)$ & $-0.0061(12)$ \\
O11C & $0.044(2)$ & $0.0294(19)$ & $0.0392(19)$ & $-0.0148(16)$ & $-0.0004(16)$ & $-0.0138(15)$ \\
O12A & $0.0244(16)$ & $0.0355(18)$ & $0.0277(16)$ & $-0.0105(14)$ & $-0.0123(13)$ & $0.0103(14)$ \\
O12B & $0.0364(18)$ & $0.0329(18)$ & $0.0211(15)$ & $-0.0207(15)$ & $-0.0009(13)$ & $-0.0038(13)$ \\
O12C & $0.0249(17)$ & $0.0214(17)$ & $0.047(2)$ & $-0.0014(13)$ & $-0.0095(15)$ & $0.0120(15)$ \\
N4A & $0.042(3)$ & $0.062(3)$ & $0.030(2)$ & $-0.030(3)$ & $-0.010(2)$ & $0.015(2)$ \\
N4B & $0.049(3)$ & $0.022(2)$ & $0.030(2)$ & $-0.013(2)$ & $-0.009(2)$ & $-0.0008(17)$ \\
N4C & $0.028(2)$ & $0.020(2)$ & $0.061(3)$ & $-0.0033(18)$ & $0.002(2)$ & $0.005(2)$ \\
C1A & $0.024(2)$ & $0.020(2)$ & $0.017(2)$ & $-0.0060(18)$ & $-0.0057(17)$ & $-0.0019(17)$ \\
C1B & $0.028(2)$ & $0.021(2)$ & $0.0153(19)$ & $-0.0092(19)$ & $-0.0069(17)$ & $0.0001(17)$ \\
C1C & $0.0153(19)$ & $0.016(2)$ & $0.019(2)$ & $-0.0049(16)$ & $-0.0023(16)$ & $-0.0001(16)$ \\
C2A & $0.024(2)$ & $0.028(2)$ & $0.028(2)$ & $-0.0071(19)$ & $-0.0114(19)$ & $0.0045(19)$ \\
C2B & $0.025(2)$ & $0.025(2)$ & $0.021(2)$ & $-0.0074(19)$ & $-0.0033(18)$ & $0.0024(18)$
\end{tabular}




\begin{tabular}{lllllll} 
& & & & & \\
C2C & $0.026(2)$ & $0.022(2)$ & $0.026(2)$ & $-0.0041(19)$ & $-0.0133(18)$ & $-0.0007(18)$ \\
C3A & $0.036(3)$ & $0.028(3)$ & $0.027(2)$ & $-0.013(2)$ & $-0.014(2)$ & $0.011(2)$ \\
C3B & $0.031(2)$ & $0.021(2)$ & $0.022(2)$ & $-0.0007(19)$ & $-0.0041(18)$ & $-0.0032(18)$ \\
C3C & $0.033(3)$ & $0.024(2)$ & $0.032(2)$ & $-0.007(2)$ & $-0.010(2)$ & $-0.010(2)$ \\
C4A & $0.034(3)$ & $0.034(3)$ & $0.020(2)$ & $-0.021(2)$ & $-0.0077(19)$ & $0.0017(19)$ \\
C4B & $0.040(3)$ & $0.016(2)$ & $0.015(2)$ & $-0.0080(19)$ & $-0.0080(18)$ & $0.0036(17)$ \\
C4C & $0.020(2)$ & $0.013(2)$ & $0.038(3)$ & $-0.0043(18)$ & $0.0024(19)$ & $0.0033(19)$ \\
C5A & $0.019(2)$ & $0.045(3)$ & $0.023(2)$ & $-0.012(2)$ & $-0.0061(18)$ & $-0.004(2)$ \\
C5B & $0.032(2)$ & $0.026(2)$ & $0.022(2)$ & $-0.013(2)$ & $-0.0057(19)$ & $0.0028(19)$ \\
C5C & $0.030(2)$ & $0.027(3)$ & $0.029(2)$ & $-0.001(2)$ & $-0.012(2)$ & $0.011(2)$ \\
C6A & $0.028(2)$ & $0.026(2)$ & $0.018(2)$ & $-0.0065(19)$ & $-0.0082(18)$ & $0.0033(18)$ \\
C6B & $0.031(2)$ & $0.018(2)$ & $0.021(2)$ & $-0.0050(19)$ & $-0.0052(18)$ & $0.0007(17)$ \\
C6C & $0.030(2)$ & $0.032(3)$ & $0.022(2)$ & $-0.007(2)$ & $-0.0127(19)$ & $0.0006(19)$ \\
C11A & $0.026(2)$ & $0.020(2)$ & $0.018(2)$ & $-0.0092(18)$ & $-0.0077(17)$ & $0.0001(17)$ \\
C11B & $0.027(2)$ & $0.024(2)$ & $0.021(2)$ & $-0.0107(19)$ & $-0.0117(18)$ & $0.0001(18)$ \\
C11C & $0.019(2)$ & $0.019(2)$ & $0.021(2)$ & $-0.0110(18)$ & $0.0039(17)$ & $0.0000(17)$ \\
O3W & $0.031(2)$ & $0.071(3)$ & $0.0254(18)$ & $-0.0003(19)$ & $-0.0093(15)$ & $0.0010(18)$ \\
& & & & & & \\
\hline
\end{tabular}

Geometric parameters $\left(A,{ }^{\circ}\right)$

\begin{tabular}{|c|c|c|c|}
\hline Er1-O1W & $2.373(2)$ & $\mathrm{C} 1 \mathrm{~A}-\mathrm{C} 2 \mathrm{~A}$ & $1.391(6)$ \\
\hline $\mathrm{Er} 1-\mathrm{O} 2 \mathrm{~W}$ & $2.295(3)$ & $\mathrm{C} 1 \mathrm{~B}-\mathrm{C} 2 \mathrm{~B}$ & $1.393(6)$ \\
\hline $\mathrm{Er} 1-\mathrm{O} 11 \mathrm{~A}$ & $2.477(3)$ & $\mathrm{C} 1 \mathrm{~B}-\mathrm{C} 6 \mathrm{~B}$ & $1.393(7)$ \\
\hline Er1-O11B & $2.478(3)$ & $\mathrm{C} 1 \mathrm{~B}-\mathrm{C} 11 \mathrm{~B}$ & $1.480(6)$ \\
\hline $\mathrm{Er} 1-\mathrm{O} 12 \mathrm{~A}$ & $2.333(3)$ & $\mathrm{C} 1 \mathrm{C}-\mathrm{C} 11 \mathrm{C}$ & $1.490(6)$ \\
\hline $\mathrm{Er} 1-\mathrm{O} 12 \mathrm{~B}$ & $2.385(3)$ & $\mathrm{C} 1 \mathrm{C}-\mathrm{C} 6 \mathrm{C}$ & $1.380(6)$ \\
\hline $\mathrm{Er} 1-\mathrm{O} 12 \mathrm{C}$ & $2.232(3)$ & $\mathrm{C} 1 \mathrm{C}-\mathrm{C} 2 \mathrm{C}$ & $1.390(6)$ \\
\hline Er1-O11C & $2.233(4)$ & $\mathrm{C} 2 \mathrm{~A}-\mathrm{C} 3 \mathrm{~A}$ & $1.362(6)$ \\
\hline $\mathrm{O} 11 \mathrm{~A}-\mathrm{C} 11 \mathrm{~A}$ & $1.257(5)$ & $\mathrm{C} 2 \mathrm{~B}-\mathrm{C} 3 \mathrm{~B}$ & $1.375(6)$ \\
\hline $\mathrm{O} 11 \mathrm{~B}-\mathrm{C} 11 \mathrm{~B}$ & $1.262(5)$ & $\mathrm{C} 2 \mathrm{C}-\mathrm{C} 3 \mathrm{C}$ & $1.374(6)$ \\
\hline $\mathrm{O} 11 \mathrm{C}-\mathrm{C} 11 \mathrm{C}$ & $1.245(6)$ & $\mathrm{C} 3 \mathrm{~A}-\mathrm{C} 4 \mathrm{~A}$ & $1.397(7)$ \\
\hline $\mathrm{O} 12 \mathrm{~A}-\mathrm{C} 11 \mathrm{~A}$ & $1.273(6)$ & $\mathrm{C} 3 \mathrm{~B}-\mathrm{C} 4 \mathrm{~B}$ & $1.388(7)$ \\
\hline $\mathrm{O} 12 \mathrm{~B}-\mathrm{C} 11 \mathrm{~B}$ & $1.273(6)$ & $\mathrm{C} 3 \mathrm{C}-\mathrm{C} 4 \mathrm{C}$ & $1.379(6)$ \\
\hline $\mathrm{O} 12 \mathrm{C}-\mathrm{C} 11 \mathrm{C}$ & $1.254(5)$ & $\mathrm{C} 4 \mathrm{~A}-\mathrm{C} 5 \mathrm{~A}$ & $1.402(6)$ \\
\hline $\mathrm{O} 1 \mathrm{~W}-\mathrm{H} 12 \mathrm{~W}$ & $0.82(3)$ & $\mathrm{C} 4 \mathrm{~B}-\mathrm{C} 5 \mathrm{~B}$ & $1.386(6)$ \\
\hline $\mathrm{O} 1 \mathrm{~W}-\mathrm{H} 11 \mathrm{~W}$ & $0.82(4)$ & $\mathrm{C} 4 \mathrm{C}-\mathrm{C} 5 \mathrm{C}$ & $1.391(6)$ \\
\hline $\mathrm{O} 2 \mathrm{~W}-\mathrm{H} 21 \mathrm{~W}$ & $0.84(4)$ & $\mathrm{C} 5 \mathrm{~A}-\mathrm{C} 6 \mathrm{~A}$ & $1.382(6)$ \\
\hline $\mathrm{O} 2 \mathrm{~W}-\mathrm{H} 22 \mathrm{~W}$ & $0.86(4)$ & $\mathrm{C} 5 \mathrm{~B}-\mathrm{C} 6 \mathrm{~B}$ & $1.383(6)$ \\
\hline $\mathrm{O} 3 \mathrm{~W}-\mathrm{H} 31 \mathrm{~W}$ & $0.83(4)$ & $\mathrm{C} 5 \mathrm{C}-\mathrm{C} 6 \mathrm{C}$ & $1.381(6)$ \\
\hline $\mathrm{O} 3 \mathrm{~W}-\mathrm{H} 32 \mathrm{~W}$ & $0.85(5)$ & $\mathrm{C} 2 \mathrm{~A}-\mathrm{H} 2 \mathrm{~A}$ & 0.9300 \\
\hline $\mathrm{N} 4 \mathrm{~A}-\mathrm{C} 4 \mathrm{~A}$ & $1.375(6)$ & $\mathrm{C} 2 \mathrm{~B}-\mathrm{H} 2 \mathrm{~B}$ & 0.9300 \\
\hline $\mathrm{N} 4 \mathrm{~B}-\mathrm{C} 4 \mathrm{~B}$ & $1.388(6)$ & $\mathrm{C} 2 \mathrm{C}-\mathrm{H} 2 \mathrm{C}$ & 0.9300 \\
\hline $\mathrm{N} 4 \mathrm{C}-\mathrm{C} 4 \mathrm{C}$ & $1.409(6)$ & $\mathrm{C} 3 \mathrm{~A}-\mathrm{H} 3 \mathrm{~A}$ & 0.9300 \\
\hline $\mathrm{N} 4 \mathrm{~A}-\mathrm{H} 41 \mathrm{~A}$ & $0.87(4)$ & $\mathrm{C} 3 \mathrm{~B}-\mathrm{H} 3 \mathrm{~B}$ & 0.9300 \\
\hline $\mathrm{N} 4 \mathrm{~A}-\mathrm{H} 42 \mathrm{~A}$ & $0.88(4)$ & $\mathrm{C} 3 \mathrm{C}-\mathrm{H} 3 \mathrm{C}$ & 0.9300 \\
\hline $\mathrm{N} 4 \mathrm{~B}-\mathrm{H} 41 \mathrm{~B}$ & $0.86(4)$ & $\mathrm{C} 5 \mathrm{~A}-\mathrm{H} 5 \mathrm{~A}$ & 0.9300 \\
\hline $\mathrm{N} 4 \mathrm{~B}-\mathrm{H} 42 \mathrm{~B}$ & $0.86(3)$ & $\mathrm{C} 5 \mathrm{~B}-\mathrm{H} 5 \mathrm{~B}$ & 0.9300 \\
\hline $\mathrm{N} 4 \mathrm{C}-\mathrm{H} 41 \mathrm{C}$ & $0.89(5)$ & $\mathrm{C} 5 \mathrm{C}-\mathrm{H} 5 \mathrm{C}$ & 0.9300 \\
\hline
\end{tabular}




\begin{tabular}{|c|c|c|c|}
\hline $\mathrm{N} 4 \mathrm{C}-\mathrm{H} 42 \mathrm{C}$ & $0.86(3)$ & $\mathrm{C} 6 \mathrm{~A}-\mathrm{H} 6 \mathrm{~A}$ & 0.9300 \\
\hline $\mathrm{C} 1 \mathrm{~A}-\mathrm{C} 11 \mathrm{~A}$ & $1.482(6)$ & $\mathrm{C} 6 \mathrm{~B}-\mathrm{H} 6 \mathrm{~B}$ & 0.9300 \\
\hline $\mathrm{C} 1 \mathrm{~A}-\mathrm{C} 6 \mathrm{~A}$ & $1.386(7)$ & $\mathrm{C} 6 \mathrm{C}-\mathrm{H} 6 \mathrm{C}$ & 0.9300 \\
\hline $\mathrm{O} 1 \mathrm{~W}-\mathrm{Er} 1-\mathrm{O} 2 \mathrm{~W}$ & $87.02(12)$ & $\mathrm{C} 2 \mathrm{C}-\mathrm{C} 1 \mathrm{C}-\mathrm{C} 6 \mathrm{C}$ & $118.8(4)$ \\
\hline $\mathrm{O} 1 \mathrm{~W}-\mathrm{Er} 1-\mathrm{O} 11 \mathrm{~A}$ & $131.43(9)$ & $\mathrm{C} 6 \mathrm{C}-\mathrm{C} 1 \mathrm{C}-\mathrm{C} 11 \mathrm{C}$ & $121.1(4)$ \\
\hline $\mathrm{O} 1 \mathrm{~W}-\mathrm{Er} 1-\mathrm{O} 11 \mathrm{~B}$ & $72.20(9)$ & $\mathrm{C} 1 \mathrm{~A}-\mathrm{C} 2 \mathrm{~A}-\mathrm{C} 3 \mathrm{~A}$ & $121.6(4)$ \\
\hline $\mathrm{O} 1 \mathrm{~W}-\mathrm{Er} 1-\mathrm{O} 12 \mathrm{~A}$ & $151.62(11)$ & $\mathrm{C} 1 \mathrm{~B}-\mathrm{C} 2 \mathrm{~B}-\mathrm{C} 3 \mathrm{~B}$ & $121.2(4)$ \\
\hline $\mathrm{O} 1 \mathrm{~W}-\mathrm{Er} 1-\mathrm{O} 12 \mathrm{~B}$ & $124.33(11)$ & $\mathrm{C} 1 \mathrm{C}-\mathrm{C} 2 \mathrm{C}-\mathrm{C} 3 \mathrm{C}$ & $120.6(4)$ \\
\hline $\mathrm{O} 1 \mathrm{~W}-\mathrm{Er} 1-\mathrm{O} 12 \mathrm{C}$ & $79.98(10)$ & $\mathrm{C} 2 \mathrm{~A}-\mathrm{C} 3 \mathrm{~A}-\mathrm{C} 4 \mathrm{~A}$ & $119.9(4)$ \\
\hline $\mathrm{O} 1 \mathrm{~W}-\mathrm{Er} 1-\mathrm{O} 11 \mathrm{C}^{\mathrm{i}}$ & $73.68(12)$ & $\mathrm{C} 2 \mathrm{~B}-\mathrm{C} 3 \mathrm{~B}-\mathrm{C} 4 \mathrm{~B}$ & $120.7(4)$ \\
\hline $\mathrm{O} 2 \mathrm{~W}-\mathrm{Er} 1-\mathrm{O} 11 \mathrm{~A}$ & $126.78(12)$ & $\mathrm{C} 2 \mathrm{C}-\mathrm{C} 3 \mathrm{C}-\mathrm{C} 4 \mathrm{C}$ & $120.6(4)$ \\
\hline $\mathrm{O} 2 \mathrm{~W}-\mathrm{Er} 1-\mathrm{O} 11 \mathrm{~B}$ & $78.50(12)$ & $\mathrm{C} 3 \mathrm{~A}-\mathrm{C} 4 \mathrm{~A}-\mathrm{C} 5 \mathrm{~A}$ & $119.1(4)$ \\
\hline $\mathrm{O} 2 \mathrm{~W}-\mathrm{Er} 1-\mathrm{O} 12 \mathrm{~A}$ & $75.02(12)$ & $\mathrm{N} 4 \mathrm{~A}-\mathrm{C} 4 \mathrm{~A}-\mathrm{C} 5 \mathrm{~A}$ & $121.4(4)$ \\
\hline $\mathrm{O} 2 \mathrm{~W}-\mathrm{Er} 1-\mathrm{O} 12 \mathrm{~B}$ & $93.16(12)$ & $\mathrm{N} 4 \mathrm{~A}-\mathrm{C} 4 \mathrm{~A}-\mathrm{C} 3 \mathrm{~A}$ & $119.5(4)$ \\
\hline $\mathrm{O} 2 \mathrm{~W}-\mathrm{Er} 1-\mathrm{O} 12 \mathrm{C}$ & $156.11(12)$ & $\mathrm{C} 3 \mathrm{~B}-\mathrm{C} 4 \mathrm{~B}-\mathrm{C} 5 \mathrm{~B}$ & $118.7(4)$ \\
\hline $\mathrm{O} 2 \mathrm{~W}-\mathrm{Er} 1-\mathrm{O} 11 \mathrm{C}^{\mathrm{i}}$ & $85.80(13)$ & $\mathrm{N} 4 \mathrm{~B}-\mathrm{C} 4 \mathrm{~B}-\mathrm{C} 5 \mathrm{~B}$ & $120.5(4)$ \\
\hline $\mathrm{O} 11 \mathrm{~A}-\mathrm{Er} 1-\mathrm{O} 11 \mathrm{~B}$ & $140.04(10)$ & $\mathrm{N} 4 \mathrm{~B}-\mathrm{C} 4 \mathrm{~B}-\mathrm{C} 3 \mathrm{~B}$ & $120.8(4)$ \\
\hline $\mathrm{O} 11 \mathrm{~A}-\mathrm{Er} 1-\mathrm{O} 12 \mathrm{~A}$ & $53.86(10)$ & $\mathrm{C} 3 \mathrm{C}-\mathrm{C} 4 \mathrm{C}-\mathrm{C} 5 \mathrm{C}$ & $118.9(4)$ \\
\hline $\mathrm{O} 11 \mathrm{~A}-\mathrm{Er} 1-\mathrm{O} 12 \mathrm{~B}$ & $91.09(11)$ & $\mathrm{N} 4 \mathrm{C}-\mathrm{C} 4 \mathrm{C}-\mathrm{C} 3 \mathrm{C}$ & $121.9(4)$ \\
\hline $\mathrm{O} 11 \mathrm{~A}-\mathrm{Er} 1-\mathrm{O} 12 \mathrm{C}$ & $76.09(10)$ & $\mathrm{N} 4 \mathrm{C}-\mathrm{C} 4 \mathrm{C}-\mathrm{C} 5 \mathrm{C}$ & $119.2(4)$ \\
\hline $\mathrm{O} 11 \mathrm{~A}-\mathrm{Er} 1-\mathrm{O} 11 \mathrm{C}^{\mathrm{i}}$ & $75.35(12)$ & $\mathrm{C} 4 \mathrm{~A}-\mathrm{C} 5 \mathrm{~A}-\mathrm{C} 6 \mathrm{~A}$ & $119.8(4)$ \\
\hline $\mathrm{O} 11 \mathrm{~B}-\mathrm{Er} 1-\mathrm{O} 12 \mathrm{~A}$ & $123.63(9)$ & $\mathrm{C} 4 \mathrm{~B}-\mathrm{C} 5 \mathrm{~B}-\mathrm{C} 6 \mathrm{~B}$ & $120.6(4)$ \\
\hline $\mathrm{O} 11 \mathrm{~B}-\mathrm{Er} 1-\mathrm{O} 12 \mathrm{~B}$ & $53.56(10)$ & $\mathrm{C} 4 \mathrm{C}-\mathrm{C} 5 \mathrm{C}-\mathrm{C} 6 \mathrm{C}$ & $120.3(4)$ \\
\hline $\mathrm{O} 11 \mathrm{~B}-\mathrm{E} 1-\mathrm{O} 12 \mathrm{C}$ & $78.48(10)$ & $\mathrm{C} 1 \mathrm{~A}-\mathrm{C} 6 \mathrm{~A}-\mathrm{C} 5 \mathrm{~A}$ & $120.8(4)$ \\
\hline $\mathrm{O} 11 \mathrm{~B}-\mathrm{Er} 1-\mathrm{O} 11 \mathrm{C}^{\mathrm{i}}$ & $142.95(11)$ & $\mathrm{C} 1 \mathrm{~B}-\mathrm{C} 6 \mathrm{~B}-\mathrm{C} 5 \mathrm{~B}$ & $120.8(4)$ \\
\hline $\mathrm{O} 12 \mathrm{~A}-\mathrm{Er} 1-\mathrm{O} 12 \mathrm{~B}$ & $79.21(10)$ & $\mathrm{C} 1 \mathrm{C}-\mathrm{C} 6 \mathrm{C}-\mathrm{C} 5 \mathrm{C}$ & $120.6(4)$ \\
\hline $\mathrm{O} 12 \mathrm{~A}-\mathrm{Er} 1-\mathrm{O} 12 \mathrm{C}$ & $123.94(10)$ & $\mathrm{O} 11 \mathrm{~A}-\mathrm{C} 11 \mathrm{~A}-\mathrm{C} 1 \mathrm{~A}$ & $122.2(4)$ \\
\hline $\mathrm{O} 11 \mathrm{C}-\mathrm{Er} 1-\mathrm{O} 12 \mathrm{~A}$ & $83.11(12)$ & $\mathrm{O} 12 \mathrm{~A}-\mathrm{C} 11 \mathrm{~A}-\mathrm{C} 1 \mathrm{~A}$ & $118.5(4)$ \\
\hline $\mathrm{O} 12 \mathrm{~B}-\mathrm{E} 1-\mathrm{O} 12 \mathrm{C}$ & $78.15(10)$ & $\mathrm{O} 11 \mathrm{~A}-\mathrm{C} 11 \mathrm{~A}-\mathrm{O} 12 \mathrm{~A}$ & $119.2(4)$ \\
\hline 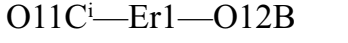 & $161.93(12)$ & $\mathrm{O} 11 \mathrm{~B}-\mathrm{C} 11 \mathrm{~B}-\mathrm{C} 1 \mathrm{~B}$ & $120.7(4)$ \\
\hline $\mathrm{O} 11 \mathrm{C}-\mathrm{E} 1-\mathrm{O} 12 \mathrm{C}$ & $109.26(11)$ & $\mathrm{O} 12 \mathrm{~B}-\mathrm{C} 11 \mathrm{~B}-\mathrm{C} 1 \mathrm{~B}$ & $119.4(3)$ \\
\hline $\mathrm{Er} 1-\mathrm{O} 11 \mathrm{~A}-\mathrm{C} 11 \mathrm{~A}$ & $90.0(3)$ & $\mathrm{O} 11 \mathrm{~B}-\mathrm{C} 11 \mathrm{~B}-\mathrm{O} 12 \mathrm{~B}$ & $119.8(4)$ \\
\hline $\mathrm{Er} 1-\mathrm{O} 11 \mathrm{~B}-\mathrm{C} 11 \mathrm{~B}$ & $90.2(3)$ & $\mathrm{O} 11 \mathrm{C}-\mathrm{C} 11 \mathrm{C}-\mathrm{O} 12 \mathrm{C}$ & $124.0(4)$ \\
\hline $\mathrm{Er} 1^{\mathrm{i}}-\mathrm{O} 11 \mathrm{C}-\mathrm{C} 11 \mathrm{C}$ & $165.0(3)$ & $\mathrm{O} 11 \mathrm{C}-\mathrm{C} 11 \mathrm{C}-\mathrm{C} 1 \mathrm{C}$ & $117.9(4)$ \\
\hline $\mathrm{Er} 1-\mathrm{O} 12 \mathrm{~A}-\mathrm{C} 11 \mathrm{~A}$ & $96.3(2)$ & $\mathrm{O} 12 \mathrm{C}-\mathrm{C} 11 \mathrm{C}-\mathrm{C} 1 \mathrm{C}$ & $118.1(4)$ \\
\hline $\mathrm{Er} 1-\mathrm{O} 12 \mathrm{~B}-\mathrm{C} 11 \mathrm{~B}$ & $94.2(2)$ & $\mathrm{C} 1 \mathrm{~A}-\mathrm{C} 2 \mathrm{~A}-\mathrm{H} 2 \mathrm{~A}$ & 119.00 \\
\hline $\mathrm{Er} 1-\mathrm{O} 12 \mathrm{C}-\mathrm{C} 11 \mathrm{C}$ & $138.1(3)$ & $\mathrm{C} 3 \mathrm{~A}-\mathrm{C} 2 \mathrm{~A}-\mathrm{H} 2 \mathrm{~A}$ & 119.00 \\
\hline $\mathrm{H} 11 \mathrm{~W}-\mathrm{O} 1 \mathrm{~W}-\mathrm{H} 12 \mathrm{~W}$ & $100(4)$ & $\mathrm{C} 3 \mathrm{~B}-\mathrm{C} 2 \mathrm{~B}-\mathrm{H} 2 \mathrm{~B}$ & 119.00 \\
\hline $\mathrm{Er} 1-\mathrm{O} 1 \mathrm{~W}-\mathrm{H} 11 \mathrm{~W}$ & $119(3)$ & $\mathrm{C} 1 \mathrm{~B}-\mathrm{C} 2 \mathrm{~B}-\mathrm{H} 2 \mathrm{~B}$ & 119.00 \\
\hline $\mathrm{Er} 1-\mathrm{O} 1 \mathrm{~W}-\mathrm{H} 12 \mathrm{~W}$ & $141(2)$ & $\mathrm{C} 1 \mathrm{C}-\mathrm{C} 2 \mathrm{C}-\mathrm{H} 2 \mathrm{C}$ & 120.00 \\
\hline $\mathrm{H} 21 \mathrm{~W}-\mathrm{O} 2 \mathrm{~W}-\mathrm{H} 22 \mathrm{~W}$ & $107(5)$ & $\mathrm{C} 3 \mathrm{C}-\mathrm{C} 2 \mathrm{C}-\mathrm{H} 2 \mathrm{C}$ & 120.00 \\
\hline $\mathrm{Er} 1-\mathrm{O} 2 \mathrm{~W}-\mathrm{H} 21 \mathrm{~W}$ & $122(4)$ & $\mathrm{C} 4 \mathrm{~A}-\mathrm{C} 3 \mathrm{~A}-\mathrm{H} 3 \mathrm{~A}$ & 120.00 \\
\hline $\mathrm{Er} 1-\mathrm{O} 2 \mathrm{~W}-\mathrm{H} 22 \mathrm{~W}$ & $130(3)$ & $\mathrm{C} 2 \mathrm{~A}-\mathrm{C} 3 \mathrm{~A}-\mathrm{H} 3 \mathrm{~A}$ & 120.00 \\
\hline $\mathrm{H} 31 \mathrm{~W}-\mathrm{O} 3 \mathrm{~W}-\mathrm{H} 32 \mathrm{~W}$ & $104(5)$ & $\mathrm{C} 2 \mathrm{~B}-\mathrm{C} 3 \mathrm{~B}-\mathrm{H} 3 \mathrm{~B}$ & 120.00 \\
\hline $\mathrm{C} 4 \mathrm{~A}-\mathrm{N} 4 \mathrm{~A}-\mathrm{H} 41 \mathrm{~A}$ & $121(4)$ & $\mathrm{C} 4 \mathrm{~B}-\mathrm{C} 3 \mathrm{~B}-\mathrm{H} 3 \mathrm{~B}$ & 120.00 \\
\hline $\mathrm{H} 41 \mathrm{~A}-\mathrm{N} 4 \mathrm{~A}-\mathrm{H} 42 \mathrm{~A}$ & $122(5)$ & $\mathrm{C} 4 \mathrm{C}-\mathrm{C} 3 \mathrm{C}-\mathrm{H} 3 \mathrm{C}$ & 120.00 \\
\hline $\mathrm{C} 4 \mathrm{~A}-\mathrm{N} 4 \mathrm{~A}-\mathrm{H} 42 \mathrm{~A}$ & $115(3)$ & $\mathrm{C} 2 \mathrm{C}-\mathrm{C} 3 \mathrm{C}-\mathrm{H} 3 \mathrm{C}$ & 120.00 \\
\hline
\end{tabular}




\begin{tabular}{|c|c|}
\hline $\mathrm{C} 4 \mathrm{~B}-\mathrm{N} 4 \mathrm{~B}-\mathrm{H} 42 \mathrm{~B}$ & $111(4)$ \\
\hline $\mathrm{H} 41 \mathrm{~B}-\mathrm{N} 4 \mathrm{~B}-\mathrm{H} 42 \mathrm{~B}$ & $112(4)$ \\
\hline $\mathrm{C} 4 \mathrm{~B}-\mathrm{N} 4 \mathrm{~B}-\mathrm{H} 41 \mathrm{~B}$ & $116(3)$ \\
\hline $\mathrm{C} 4 \mathrm{C}-\mathrm{N} 4 \mathrm{C}-\mathrm{H} 41 \mathrm{C}$ & $108(3)$ \\
\hline $\mathrm{H} 41 \mathrm{C}-\mathrm{N} 4 \mathrm{C}-\mathrm{H} 42 \mathrm{C}$ & $121(5)$ \\
\hline $\mathrm{C} 4 \mathrm{C}-\mathrm{N} 4 \mathrm{C}-\mathrm{H} 42 \mathrm{C}$ & $110(3)$ \\
\hline $\mathrm{C} 2 \mathrm{~A}-\mathrm{C} 1 \mathrm{~A}-\mathrm{C} 6 \mathrm{~A}$ & $118.6(4)$ \\
\hline $\mathrm{C} 6 \mathrm{~A}-\mathrm{C} 1 \mathrm{~A}-\mathrm{C} 11 \mathrm{~A}$ & $121.7(4)$ \\
\hline $\mathrm{C} 2 \mathrm{~A}-\mathrm{C} 1 \mathrm{~A}-\mathrm{C} 11 \mathrm{~A}$ & $119.7(4)$ \\
\hline $\mathrm{C} 2 \mathrm{~B}-\mathrm{C} 1 \mathrm{~B}-\mathrm{C} 11 \mathrm{~B}$ & $120.6(4)$ \\
\hline $\mathrm{C} 6 \mathrm{~B}-\mathrm{C} 1 \mathrm{~B}-\mathrm{C} 11 \mathrm{~B}$ & $121.3(4)$ \\
\hline $\mathrm{C} 2 \mathrm{~B}-\mathrm{C} 1 \mathrm{~B}-\mathrm{C} 6 \mathrm{~B}$ & $118.0(4)$ \\
\hline $\mathrm{C} 2 \mathrm{C}-\mathrm{C} 1 \mathrm{C}-\mathrm{C} 11 \mathrm{C}$ & $120.1(4)$ \\
\hline $\mathrm{O} 1 \mathrm{~W}-\mathrm{Er} 1-\mathrm{O} 11 \mathrm{~A}-\mathrm{C} 11 \mathrm{~A}$ & $139.1(2)$ \\
\hline $\mathrm{O} 2 \mathrm{~W}-\mathrm{Er} 1-\mathrm{O} 11 \mathrm{~A}-\mathrm{C} 11 \mathrm{~A}$ & $14.1(3)$ \\
\hline $\mathrm{O} 11 \mathrm{~B}-\mathrm{Er} 1-\mathrm{O} 11 \mathrm{~A}-\mathrm{C} 11 \mathrm{~A}$ & $-106.2(3)$ \\
\hline $\mathrm{O} 12 \mathrm{~A}-\mathrm{Er} 1-\mathrm{O} 11 \mathrm{~A}-\mathrm{C} 11 \mathrm{~A}$ & $-4.9(2)$ \\
\hline $\mathrm{O} 12 \mathrm{~B}-\mathrm{Er} 1-\mathrm{O} 11 \mathrm{~A}-\mathrm{C} 11 \mathrm{~A}$ & $-80.7(2)$ \\
\hline $\mathrm{O} 12 \mathrm{C}-\mathrm{Er} 1-\mathrm{O} 11 \mathrm{~A}-\mathrm{C} 11 \mathrm{~A}$ & $-158.2(2)$ \\
\hline $\mathrm{O} 11 \mathrm{C}-\mathrm{Er} 1-\mathrm{O} 11 \mathrm{~A}-\mathrm{C} 11 \mathrm{~A}$ & $87.3(2)$ \\
\hline $\mathrm{O} 1 \mathrm{~W}-\mathrm{Er} 1-\mathrm{O} 11 \mathrm{~B}-\mathrm{C} 11 \mathrm{~B}$ & $158.2(3)$ \\
\hline $\mathrm{O} 2 \mathrm{~W}-\mathrm{Er} 1-\mathrm{O} 11 \mathrm{~B}-\mathrm{C} 11 \mathrm{~B}$ & $-111.2(2)$ \\
\hline $\mathrm{O} 11 \mathrm{~A}-\mathrm{Er} 1-\mathrm{O} 11 \mathrm{~B}-\mathrm{C} 11 \mathrm{~B}$ & $23.9(3)$ \\
\hline $\mathrm{O} 12 \mathrm{~A}-\mathrm{Er} 1-\mathrm{O} 11 \mathrm{~B}-\mathrm{C} 11 \mathrm{~B}$ & $-48.1(3)$ \\
\hline $\mathrm{O} 12 \mathrm{~B}-\mathrm{Er} 1-\mathrm{O} 11 \mathrm{~B}-\mathrm{C} 11 \mathrm{~B}$ & $-8.5(2)$ \\
\hline $\mathrm{O} 12 \mathrm{C}-\mathrm{Er} 1-\mathrm{O} 11 \mathrm{~B}-\mathrm{C} 11 \mathrm{~B}$ & $75.2(2)$ \\
\hline $\mathrm{O} 11 \mathrm{C}-\mathrm{i}-\mathrm{Er} 1-\mathrm{O} 11 \mathrm{~B}-\mathrm{C} 11 \mathrm{~B}$ & $-178.1(2)$ \\
\hline $\mathrm{O} 1 \mathrm{~W}-\mathrm{E} 1-\mathrm{O} 12 \mathrm{~A}-\mathrm{C} 11 \mathrm{~A}$ & $-107.0(3)$ \\
\hline $\mathrm{O} 2 \mathrm{~W}-\mathrm{Er} 1-\mathrm{O} 12 \mathrm{~A}-\mathrm{C} 11 \mathrm{~A}$ & $-159.5(3)$ \\
\hline $\mathrm{O} 11 \mathrm{~A}-\mathrm{Er} 1-\mathrm{O} 12 \mathrm{~A}-\mathrm{C} 11 \mathrm{~A}$ & $4.9(2)$ \\
\hline $\mathrm{O} 11 \mathrm{~B}-\mathrm{Er} 1-\mathrm{O} 12 \mathrm{~A}-\mathrm{C} 11 \mathrm{~A}$ & $135.7(2)$ \\
\hline $\mathrm{O} 12 \mathrm{~B}-\mathrm{Er} 1-\mathrm{O} 12 \mathrm{~A}-\mathrm{C} 11 \mathrm{~A}$ & $104.2(3)$ \\
\hline $\mathrm{O} 12 \mathrm{C}-\mathrm{Er} 1-\mathrm{O} 12 \mathrm{~A}-\mathrm{C} 11 \mathrm{~A}$ & $36.6(3)$ \\
\hline $\mathrm{O} 11 \mathrm{C}-\mathrm{E} 11-\mathrm{O} 12 \mathrm{~A}-\mathrm{C} 11 \mathrm{~A}$ & $-72.0(2)$ \\
\hline $\mathrm{O} 1 \mathrm{~W}-\mathrm{Er} 1-\mathrm{O} 12 \mathrm{~B}-\mathrm{C} 11 \mathrm{~B}$ & $-6.9(3)$ \\
\hline $\mathrm{O} 2 \mathrm{~W}-\mathrm{E} 1-\mathrm{O} 12 \mathrm{~B}-\mathrm{C} 11 \mathrm{~B}$ & $81.7(3)$ \\
\hline $\mathrm{O} 11 \mathrm{~A}-\mathrm{E} 1-\mathrm{O} 12 \mathrm{~B}-\mathrm{C} 11 \mathrm{~B}$ & $-151.4(3)$ \\
\hline $\mathrm{O} 11 \mathrm{~B}-\mathrm{E} 1-\mathrm{O} 12 \mathrm{~B}-\mathrm{C} 11 \mathrm{~B}$ & $8.5(2)$ \\
\hline $\mathrm{O} 12 \mathrm{~A}-\mathrm{Er} 1-\mathrm{O} 12 \mathrm{~B}-\mathrm{C} 11 \mathrm{~B}$ & $155.8(3)$ \\
\hline $\mathrm{O} 12 \mathrm{C}-\mathrm{Er} 1-\mathrm{O} 12 \mathrm{~B}-\mathrm{C} 11 \mathrm{~B}$ & $-75.9(3)$ \\
\hline $\mathrm{O} 1 \mathrm{~W}-\mathrm{E} 1-\mathrm{O} 12 \mathrm{C}-\mathrm{C} 11 \mathrm{C}$ & $88.7(4)$ \\
\hline $\mathrm{O} 2 \mathrm{~W}-\mathrm{Er} 1-\mathrm{O} 12 \mathrm{C}-\mathrm{C} 11 \mathrm{C}$ & $146.8(4)$ \\
\hline $\mathrm{O} 11 \mathrm{~A}-\mathrm{Er} 1-\mathrm{O} 12 \mathrm{C}-\mathrm{C} 11 \mathrm{C}$ & $-48.7(4)$ \\
\hline $\mathrm{O} 11 \mathrm{~B}-\mathrm{Er} 1-\mathrm{O} 12 \mathrm{C}-\mathrm{C} 11 \mathrm{C}$ & $162.4(4)$ \\
\hline $\mathrm{O} 12 \mathrm{~A}-\mathrm{Er} 1-\mathrm{O} 12 \mathrm{C}-\mathrm{C} 11 \mathrm{C}$ & $-74.6(4)$ \\
\hline $\mathrm{O} 12 \mathrm{~B}-\mathrm{Er} 1-\mathrm{O} 12 \mathrm{C}-\mathrm{C} 11 \mathrm{C}$ & $-142.8(4)$ \\
\hline $\mathrm{O} 11 \mathrm{C}-\mathrm{Er} 1-\mathrm{O} 12 \mathrm{C}-\mathrm{C} 11 \mathrm{C}$ & $20.1(4)$ \\
\hline
\end{tabular}

$\begin{array}{ll}\mathrm{C} 4 \mathrm{~A}-\mathrm{C} 5 \mathrm{~A}-\mathrm{H} 5 \mathrm{~A} & 120.00 \\ \mathrm{C} 6 \mathrm{~A}-\mathrm{C} 5 \mathrm{~A}-\mathrm{H} 5 \mathrm{~A} & 120.00 \\ \mathrm{C} 6 \mathrm{~B}-\mathrm{C} 5 \mathrm{~B}-\mathrm{H} 5 \mathrm{~B} & 120.00 \\ \mathrm{C} 4 \mathrm{~B}-\mathrm{C} 5 \mathrm{~B}-\mathrm{H} 5 \mathrm{~B} & 120.00 \\ \mathrm{C} 4 \mathrm{C}-\mathrm{C} 5 \mathrm{C}-\mathrm{H} 5 \mathrm{C} & 120.00 \\ \mathrm{C} 6 \mathrm{C}-\mathrm{C} 5 \mathrm{C}-\mathrm{H} 5 \mathrm{C} & 120.00 \\ \mathrm{C} 5 \mathrm{~A}-\mathrm{C} 6 \mathrm{~A}-\mathrm{H} 6 \mathrm{~A} & 120.00 \\ \mathrm{C} 1 \mathrm{~A}-\mathrm{C} 6 \mathrm{~A}-\mathrm{H} 6 \mathrm{~A} & 120.00 \\ \mathrm{C} 1 \mathrm{~B}-\mathrm{C} 6 \mathrm{~B}-\mathrm{H} 6 \mathrm{~B} & 120.00 \\ \mathrm{C} 5 \mathrm{~B}-\mathrm{C} 6 \mathrm{~B}-\mathrm{H} 6 \mathrm{~B} & 120.00 \\ \mathrm{C} 1 \mathrm{C}-\mathrm{C} 6 \mathrm{C}-\mathrm{H} 6 \mathrm{C} & 120.00 \\ \mathrm{C} 5 \mathrm{C}-\mathrm{C} 6 \mathrm{C}-\mathrm{H} 6 \mathrm{C} & 120.00\end{array}$

$\mathrm{Er} 1-\mathrm{O} 12 \mathrm{C}-\mathrm{C} 11 \mathrm{C}-\mathrm{C} 1 \mathrm{C} \quad 153.5$ (3)

$\mathrm{C} 2 \mathrm{~A}-\mathrm{C} 1 \mathrm{~A}-\mathrm{C} 6 \mathrm{~A}-\mathrm{C} 5 \mathrm{~A} \quad 1.9(6)$

$\mathrm{C} 6 \mathrm{~A}-\mathrm{C} 1 \mathrm{~A}-\mathrm{C} 2 \mathrm{~A}-\mathrm{C} 3 \mathrm{~A} \quad-1.8(6)$

$\mathrm{C} 11 \mathrm{~A}-\mathrm{C} 1 \mathrm{~A}-\mathrm{C} 2 \mathrm{~A}-\mathrm{C} 3 \mathrm{~A} \quad 176.4$ (4)

$\mathrm{C} 6 \mathrm{~A}-\mathrm{C} 1 \mathrm{~A}-\mathrm{C} 11 \mathrm{~A}-\mathrm{O} 11 \mathrm{~A} \quad-10.6(6)$

$\mathrm{C} 6 \mathrm{~A}-\mathrm{C} 1 \mathrm{~A}-\mathrm{C} 11 \mathrm{~A}-\mathrm{O} 12 \mathrm{~A} \quad 170.5$ (4)

$\mathrm{C} 11 \mathrm{~A}-\mathrm{C} 1 \mathrm{~A}-\mathrm{C} 6 \mathrm{~A}-\mathrm{C} 5 \mathrm{~A} \quad-176.3$ (4)

$\mathrm{C} 2 \mathrm{~A}-\mathrm{C} 1 \mathrm{~A}-\mathrm{C} 11 \mathrm{~A}-\mathrm{O} 11 \mathrm{~A} \quad 171.2(4)$

$\mathrm{C} 2 \mathrm{~A}-\mathrm{C} 1 \mathrm{~A}-\mathrm{C} 11 \mathrm{~A}-\mathrm{O} 12 \mathrm{~A}-7.7(6)$

$\mathrm{C} 6 \mathrm{~B}-\mathrm{C} 1 \mathrm{~B}-\mathrm{C} 2 \mathrm{~B}-\mathrm{C} 3 \mathrm{~B} \quad 2.1(6)$

$\mathrm{C} 11 \mathrm{~B}-\mathrm{C} 1 \mathrm{~B}-\mathrm{C} 2 \mathrm{~B}-\mathrm{C} 3 \mathrm{~B} \quad-174.4$ (4)

$\mathrm{C} 2 \mathrm{~B}-\mathrm{C} 1 \mathrm{~B}-\mathrm{C} 6 \mathrm{~B}-\mathrm{C} 5 \mathrm{~B} \quad-0.5(6)$

$\mathrm{C} 2 \mathrm{~B}-\mathrm{C} 1 \mathrm{~B}-\mathrm{C} 11 \mathrm{~B}-\mathrm{O} 11 \mathrm{~B} \quad 155.9$ (4)

$\mathrm{C} 2 \mathrm{~B}-\mathrm{C} 1 \mathrm{~B}-\mathrm{C} 11 \mathrm{~B}-\mathrm{O} 12 \mathrm{~B}-19.8(6)$

$\mathrm{C} 6 \mathrm{~B}-\mathrm{C} 1 \mathrm{~B}-\mathrm{C} 11 \mathrm{~B}-\mathrm{O} 11 \mathrm{~B} \quad-20.5(6)$

$\mathrm{C} 6 \mathrm{~B}-\mathrm{C} 1 \mathrm{~B}-\mathrm{C} 11 \mathrm{~B}-\mathrm{O} 12 \mathrm{~B} \quad 163.9$ (4)

$\mathrm{C} 11 \mathrm{~B}-\mathrm{C} 1 \mathrm{~B}-\mathrm{C} 6 \mathrm{~B}-\mathrm{C} 5 \mathrm{~B} \quad 176.0$ (4)

$\mathrm{C} 6 \mathrm{C}-\mathrm{C} 1 \mathrm{C}-\mathrm{C} 2 \mathrm{C}-\mathrm{C} 3 \mathrm{C} \quad-1.7(6)$

$\mathrm{C} 11 \mathrm{C}-\mathrm{C} 1 \mathrm{C}-\mathrm{C} 2 \mathrm{C}-\mathrm{C} 3 \mathrm{C} \quad 176.5(4)$

$\mathrm{C} 2 \mathrm{C}-\mathrm{C} 1 \mathrm{C}-\mathrm{C} 11 \mathrm{C}-\mathrm{O} 12 \mathrm{C} \quad-5.8(6)$

$\mathrm{C} 6 \mathrm{C}-\mathrm{C} 1 \mathrm{C}-\mathrm{C} 11 \mathrm{C}-\mathrm{O} 11 \mathrm{C} \quad-7.1(6)$

$\mathrm{C} 6 \mathrm{C}-\mathrm{C} 1 \mathrm{C}-\mathrm{C} 11 \mathrm{C}-\mathrm{O} 12 \mathrm{C} \quad 172.3$ (4)

$\mathrm{C} 2 \mathrm{C}-\mathrm{C} 1 \mathrm{C}-\mathrm{C} 6 \mathrm{C}-\mathrm{C} 5 \mathrm{C} \quad 2.2(6)$

$\mathrm{C} 11 \mathrm{C}-\mathrm{C} 1 \mathrm{C}-\mathrm{C} 6 \mathrm{C}-\mathrm{C} 5 \mathrm{C} \quad-176.0(4)$

$\mathrm{C} 2 \mathrm{C}-\mathrm{C} 1 \mathrm{C}-\mathrm{C} 11 \mathrm{C}-\mathrm{O} 11 \mathrm{C} \quad 174.8$ (4)

$\mathrm{C} 1 \mathrm{~A}-\mathrm{C} 2 \mathrm{~A}-\mathrm{C} 3 \mathrm{~A}-\mathrm{C} 4 \mathrm{~A} \quad-1.0(6)$

$\mathrm{C} 1 \mathrm{~B}-\mathrm{C} 2 \mathrm{~B}-\mathrm{C} 3 \mathrm{~B}-\mathrm{C} 4 \mathrm{~B} \quad-1.8(6)$

$\mathrm{C} 1 \mathrm{C}-\mathrm{C} 2 \mathrm{C}-\mathrm{C} 3 \mathrm{C}-\mathrm{C} 4 \mathrm{C} \quad-1.2(7)$

$\mathrm{C} 2 \mathrm{~A}-\mathrm{C} 3 \mathrm{~A}-\mathrm{C} 4 \mathrm{~A}-\mathrm{C} 5 \mathrm{~A} \quad 3.7(6)$

$\mathrm{C} 2 \mathrm{~A}-\mathrm{C} 3 \mathrm{~A}-\mathrm{C} 4 \mathrm{~A}-\mathrm{N} 4 \mathrm{~A} \quad-177.0(4)$

$\mathrm{C} 2 \mathrm{~B}-\mathrm{C} 3 \mathrm{~B}-\mathrm{C} 4 \mathrm{~B}-\mathrm{C} 5 \mathrm{~B} \quad-0.3(6)$

$\mathrm{C} 2 \mathrm{~B}-\mathrm{C} 3 \mathrm{~B}-\mathrm{C} 4 \mathrm{~B}-\mathrm{N} 4 \mathrm{~B} \quad 177.0$ (4)

$\mathrm{C} 2 \mathrm{C}-\mathrm{C} 3 \mathrm{C}-\mathrm{C} 4 \mathrm{C}-\mathrm{N} 4 \mathrm{C} \quad-177.8(4)$

$\mathrm{C} 2 \mathrm{C}-\mathrm{C} 3 \mathrm{C}-\mathrm{C} 4 \mathrm{C}-\mathrm{C} 5 \mathrm{C}$ 


$\begin{array}{llll}\text { Er1-O11A-C11A-O12A } & 8.3(4) & \mathrm{N} 4 \mathrm{~A}-\mathrm{C} 4 \mathrm{~A}-\mathrm{C} 5 \mathrm{~A}-\mathrm{C} 6 \mathrm{~A} & 177.1(4) \\ \text { Er1-O11A-C11A-C1A } & -170.6(3) & \mathrm{C} 3 \mathrm{~A}-\mathrm{C} 4 \mathrm{~A}-\mathrm{C} 5 \mathrm{~A}-\mathrm{C} 6 \mathrm{~A} & -3.7(6) \\ \text { Er1-O11B-C11B-O12B } & 14.9(4) & \mathrm{C} 3 \mathrm{~B}-\mathrm{C} 4 \mathrm{~B}-\mathrm{C} 5 \mathrm{~B}-\mathrm{C} 6 \mathrm{~B} & 1.9(6) \\ \text { Er1-O11B-C11B-C1B } & -160.7(4) & \mathrm{N} 4 \mathrm{~B}-\mathrm{C} 4 \mathrm{~B}-\mathrm{C} 5 \mathrm{~B}-\mathrm{C} 6 \mathrm{~B} & -175.4(4) \\ \text { Er1-O12A-C11A-O11A } & -8.9(4) & \mathrm{C} 3 \mathrm{C}-\mathrm{C} 4 \mathrm{C}-\mathrm{C} 5 \mathrm{C}-\mathrm{C} 6 \mathrm{C} & -3.1(7) \\ \text { Er1-O12A-C11A-C1A } & 170.1(3) & \mathrm{N} 4 \mathrm{C}-\mathrm{C} 4 \mathrm{C}-\mathrm{C} 5 \mathrm{C}-\mathrm{C} 6 \mathrm{C} & 178.2(4) \\ \text { Er1-O12B-C11B-O11B } & -15.5(4) & \mathrm{C} 4 \mathrm{~A}-\mathrm{C} 5 \mathrm{~A}-\mathrm{C} 6 \mathrm{~A}-\mathrm{C} 1 \mathrm{~A} & 0.9(6) \\ \text { Er1-O12B-C11B-C1B } & 160.1(3) & \mathrm{C} 4 \mathrm{~B}-\mathrm{C} 5 \mathrm{~B}-\mathrm{C} 6 \mathrm{~B}-\mathrm{C} 1 \mathrm{~B} & -1.5(6) \\ \text { Er1-O12C-C11C-O11C } & -27.1(6) & \mathrm{C} 4 \mathrm{C}-\mathrm{C} 5 \mathrm{C}-\mathrm{C} 6 \mathrm{C}-\mathrm{C} 1 \mathrm{C} & 0.2(7)\end{array}$

Symmetry code: (i) $-x+1,-y+1,-z+1$.

Hydrogen-bond geometry $\left(\AA,{ }^{\circ}\right)$

\begin{tabular}{lllll}
\hline$D-\mathrm{H} \cdots A$ & $D-\mathrm{H}$ & $\mathrm{H} \cdots A$ & $D \cdots A$ & $D-\mathrm{H}^{\cdots} A$ \\
\hline $\mathrm{O} 1 W-\mathrm{H} 11 W^{\cdots} \mathrm{O} 11 A^{\mathrm{i}}$ & $0.82(4)$ & $1.95(4)$ & $2.757(4)$ & $166(4)$ \\
$\mathrm{O} 1 W-\mathrm{H} 12 W \cdots \mathrm{O} 11 B^{\mathrm{ii}}$ & $0.82(3)$ & $1.98(3)$ & $2.777(4)$ & $163(4)$ \\
$\mathrm{O} 2 W-\mathrm{H} 21 W^{\cdots} \mathrm{N} 4 B^{\mathrm{iii}}$ & $0.84(4)$ & $2.09(4)$ & $2.902(5)$ & $162(5)$ \\
$\mathrm{O} 2 W-\mathrm{H} 22 W^{\cdots} \cdots \mathrm{N} 4 C^{\mathrm{iv}}$ & $0.86(4)$ & $1.89(4)$ & $2.735(6)$ & $168(5)$ \\
$\mathrm{O} 3 W-\mathrm{H} 31 W \cdots \mathrm{O} 12 B$ & $0.83(4)$ & $1.99(4)$ & $2.777(4)$ & $160(5)$ \\
$\mathrm{O} 3 W-\mathrm{H} 32 W \cdots \mathrm{O} 12 A^{\mathrm{v}}$ & $0.85(5)$ & $2.07(5)$ & $2.841(5)$ & $151(5)$ \\
$\mathrm{N} 4 A-\mathrm{H} 42 A \cdots \mathrm{O} 3 W^{\mathrm{vi}}$ & $0.88(4)$ & $2.08(4)$ & $2.902(6)$ & $156(4)$ \\
$\mathrm{N} 4 B-\mathrm{H} 41 B \cdots \mathrm{O} 3 W^{\mathrm{vii}}$ & $0.86(4)$ & $2.18(4)$ & $3.014(6)$ & $164(4)$ \\
$\mathrm{N} 4 C-\mathrm{H} 42 C \cdots \mathrm{O} 11 B^{\text {viii }}$ & $0.86(3)$ & $2.49(4)$ & $3.341(5)$ & $170(5)$ \\
\hline
\end{tabular}

Symmetry codes: (i) $-x+1,-y+1,-z+1$; (ii) $-x+2,-y+1,-z+1$; (iii) $x, y-1, z$; (iv) $x+1, y-1, z$; (v) $-x+1,-y+1,-z+2$; (vi) $-x,-y+1,-z+2$; (vii) $-x+1,-y+2$, $-z+2$; (viii) $-x+1,-y+2,-z+1$.

(II) Poly[hexakis $\left(\mu_{2}-4\right.$-chloro-3-nitrobenzoato- $\left.\kappa^{2} O: O O^{\prime}\right)$ bis(dimethyl sulfoxide- $\left.\kappa O\right)$ dierbium(III)]

Crystal data

$\left[\mathrm{Er}_{2}\left(\mathrm{C}_{7} \mathrm{H}_{3} \mathrm{ClNO}_{4}\right)_{6}\left(\mathrm{C}_{2} \mathrm{H}_{6} \mathrm{OS}\right)_{2}\right]$

$M_{r}=1694.10$

Triclinic, $P \overline{1}$

Hall symbol: -P 1

$a=8.2408(3) \AA$

$b=12.4040(8) \AA$

$c=15.3409(10) \AA$

$\alpha=111.443(6)^{\circ}$

$\beta=98.063(4)^{\circ}$

$\gamma=96.684(4)^{\circ}$

$V=1421.04(14) \AA^{3}$

Data collection

Oxford Diffraction Gemini-S CCD-detector diffractometer

Radiation source: fine-focus sealed tube

Graphite monochromator

Detector resolution: 16.077 pixels $\mathrm{mm}^{-1}$

$\omega$ scans

Absorption correction: multi-scan

(CrysAlis PRO; Agilent, 2013)

$T_{\min }=0.494, T_{\max }=0.980$
$Z=1$

$F(000)=826$

$D_{\mathrm{x}}=1.980 \mathrm{Mg} \mathrm{m}^{-3}$

Mo $K \alpha$ radiation, $\lambda=0.71073 \AA$

Cell parameters from 4326 reflections

$\theta=3.6-28.8^{\circ}$

$\mu=3.38 \mathrm{~mm}^{-1}$

$T=200 \mathrm{~K}$

Prism, colourless

$0.25 \times 0.12 \times 0.04 \mathrm{~mm}$

10041 measured reflections

5566 independent reflections

4814 reflections with $I>2 \sigma(I)$

$R_{\text {int }}=0.055$

$\theta_{\text {max }}=26.0^{\circ}, \theta_{\text {min }}=3.1^{\circ}$

$h=-10 \rightarrow 10$

$k=-15 \rightarrow 13$

$l=-16 \rightarrow 18$ 


\section{Refinement}

Refinement on $F^{2}$

Least-squares matrix: full

$R\left[F^{2}>2 \sigma\left(F^{2}\right)\right]=0.067$

$w R\left(F^{2}\right)=0.181$

$S=1.06$

5566 reflections

397 parameters

0 restraints

Primary atom site location: structure-invariant direct methods
Secondary atom site location: difference Fourier map

Hydrogen site location: inferred from neighbouring sites

$\mathrm{H}$-atom parameters constrained

$w=1 /\left[\sigma^{2}\left(F_{\mathrm{o}}^{2}\right)+(0.1243 P)^{2}\right]$ where $P=\left(F_{\mathrm{o}}^{2}+2 F_{\mathrm{c}}^{2}\right) / 3$

$(\Delta / \sigma)_{\max }=0.001$

$\Delta \rho_{\max }=6.83 \mathrm{e} \AA^{-3}$

$\Delta \rho_{\min }=-2.41$ e $\AA^{-3}$

Special details

Geometry. Bond distances, angles etc. have been calculated using the rounded fractional coordinates. All su's are estimated from the variances of the (full) variance-covariance matrix. The cell e.s.d.'s are taken into account in the estimation of distances, angles and torsion angles

Refinement. Refinement of $F^{2}$ against ALL reflections. The weighted $R$-factor $w R$ and goodness of fit $S$ are based on $F^{2}$, conventional $R$-factors $R$ are based on $F$, with $F$ set to zero for negative $F^{2}$. The threshold expression of $F^{2}>\sigma\left(F^{2}\right)$ is used only for calculating $R$-factors (gt) etc. and is not relevant to the choice of reflections for refinement. $R$-factors based on $F^{2}$ are statistically about twice as large as those based on $F$, and $R$ - factors based on ALL data will be even larger.

Fractional atomic coordinates and isotropic or equivalent isotropic displacement parameters $\left(\AA^{2}\right)$

\begin{tabular}{|c|c|c|c|c|}
\hline & $x$ & $y$ & $z$ & $U_{\text {iso }} * / U_{\text {eq }}$ \\
\hline Er1 & 0.24949 (4) & $0.48443(3)$ & $0.46092(2)$ & 0.0175 (1) \\
\hline $\mathrm{Cl} 4 \mathrm{~A}$ & $0.6408(5)$ & $0.7116(4)$ & 0.0335 (3) & $0.0699(16)$ \\
\hline C14B & $0.2887(4)$ & $-0.1334(3)$ & $0.0200(2)$ & $0.0627(10)$ \\
\hline $\mathrm{Cl} 4 \mathrm{C}$ & $-0.3399(4)$ & $-0.1283(2)$ & $0.5158(2)$ & $0.0452(9)$ \\
\hline $\mathrm{S} 1$ & $0.0342(3)$ & $0.4386(2)$ & $0.23184(16)$ & $0.0269(7)$ \\
\hline O11 & $0.1349(8)$ & $0.3972(6)$ & $0.2999(5)$ & 0.0294 (19) \\
\hline O11A & $0.6659(7)$ & $0.5633(6)$ & $0.4105(4)$ & 0.0250 (19) \\
\hline O11B & $0.6883(7)$ & $0.3352(5)$ & $0.4066(4)$ & $0.0256(17)$ \\
\hline $\mathrm{O} 11 \mathrm{C}$ & $0.0768(7)$ & $0.3102(6)$ & $0.4347(5)$ & $0.027(2)$ \\
\hline $\mathrm{O} 12 \mathrm{~A}$ & $0.3978(7)$ & $0.5899(6)$ & $0.3912(4)$ & 0.0259 (17) \\
\hline O12B & $0.4342(7)$ & $0.3679(5)$ & $0.4117(4)$ & 0.0239 (17) \\
\hline $\mathrm{O} 12 \mathrm{C}$ & $-0.0361(7)$ & $0.4170(5)$ & $0.5538(5)$ & 0.0231 (19) \\
\hline O31A & $0.1634(13)$ & $0.6185(12)$ & $0.0929(8)$ & $0.079(5)$ \\
\hline O31B & $-0.0284(11)$ & $0.0741(13)$ & $0.1852(10)$ & $0.128(6)$ \\
\hline $\mathrm{O} 31 \mathrm{C}$ & $-0.1757(16)$ & 0.1537 (14) & $0.7463(8)$ & $0.112(6)$ \\
\hline $\mathrm{O} 32 \mathrm{~A}$ & $0.3085(15)$ & $0.5798(10)$ & $-0.0175(7)$ & $0.075(4)$ \\
\hline O32B & $-0.0018(15)$ & $-0.0583(16)$ & $0.0725(12)$ & $0.174(7)$ \\
\hline $\mathrm{O} 32 \mathrm{C}$ & $-0.4244(12)$ & $0.0843(11)$ & $0.6745(8)$ & $0.074(4)$ \\
\hline N3A & $0.2942(15)$ & $0.6108(9)$ & $0.0664(7)$ & $0.050(4)$ \\
\hline N3B & $0.0575(12)$ & $0.0190(9)$ & $0.1417(8)$ & $0.050(3)$ \\
\hline $\mathrm{N} 3 \mathrm{C}$ & $-0.2816(13)$ & $0.1149(8)$ & $0.6759(7)$ & $0.043(3)$ \\
\hline C1A & $0.5617(11)$ & $0.6222(8)$ & $0.2856(6)$ & $0.023(2)$ \\
\hline C1B & $0.4672(11)$ & $0.1949(8)$ & $0.2879(6)$ & $0.023(3)$ \\
\hline $\mathrm{C} 1 \mathrm{C}$ & $-0.0974(10)$ & $0.2075(8)$ & $0.5005(6)$ & $0.023(3)$ \\
\hline $\mathrm{C} 2 \mathrm{~A}$ & $0.4248(11)$ & $0.6144(8)$ & $0.2190(6)$ & $0.025(3)$ \\
\hline $\mathrm{C} 2 \mathrm{~B}$ & $0.2996(11)$ & $0.1571(9)$ & $0.2529(7)$ & $0.029(3)$ \\
\hline
\end{tabular}




$\begin{array}{lllll}\text { C2C } & -0.1560(10) & 0.2099(8) & 0.5823(7) & 0.024(3) \\ \text { C3A } & 0.4480(14) & 0.6353(9) & 0.1384(7) & 0.036(3) \\ \text { C3B } & 0.2401(12) & 0.0547(9) & 0.1717(7) & 0.033(3) \\ \text { C3C } & -0.2293(12) & 0.1085(9) & 0.5859(7) & 0.031(3) \\ \text { C4A } & 0.6027(15) & 0.6725(10) & 0.1270(8) & 0.038(3) \\ \text { C4B } & 0.3490(13) & -0.0081(9) & 0.1221(7) & 0.036(3) \\ \text { C4C } & -0.2491(11) & 0.0009(8) & 0.5095(8) & 0.029(3) \\ \text { C5A } & 0.7399(13) & 0.6842(10) & 0.1966(8) & 0.038(3) \\ \text { C5B } & 0.5197(13) & 0.0283(9) & 0.1574(8) & 0.036(3) \\ \text { C5C } & -0.1928(13) & -0.0026(8) & 0.4290(8) & 0.034(3) \\ \text { C6A } & 0.7202(11) & 0.6582(9) & 0.2742(8) & 0.033(3) \\ \text { C6B } & 0.5809(12) & 0.1291(8) & 0.2402(7) & 0.028(3) \\ \text { C6C } & -0.1147(12) & 0.1003(8) & 0.4245(7) & 0.026(3) \\ \text { C11 } & 0.0742(13) & 0.3526(11) & 0.1178(7) & 0.041(4) \\ \text { C11A } & 0.5391(10) & 0.5897(7) & 0.3704(6) & 0.018(2) \\ \text { C11B } & 0.5342(10) & 0.3057(7) & 0.3743(6) & 0.018(3) \\ \text { C11C } & -0.0137(10) & 0.3191(8) & 0.4954(6) & 0.021(3) \\ \text { C12 } & -0.1761(12) & 0.3732(10) & 0.2168(7) & 0.035(3) \\ \text { H2A } & 0.31540 & 0.59490 & 0.22840 & 0.0300^{*} \\ \text { H2B } & 0.22320 & 0.20130 & 0.28450 & 0.0350^{*} \\ \text { H2C } & -0.14440 & 0.28250 & 0.63530 & 0.0280^{*} \\ \text { H5A } & 0.84870 & 0.71060 & 0.18990 & 0.0460^{*} \\ \text { H5B } & 0.59520 & -0.01580 & 0.12480 & 0.0430^{*} \\ \text { H5C } & -0.20710 & -0.07540 & 0.37600 & 0.0400^{*} \\ \text { H6A } & 0.81490 & 0.66480 & 0.32030 & 0.0400^{*} \\ \text { H6B } & 0.69750 & 0.15340 & 0.26440 & 0.0340^{*} \\ \text { H6C } & -0.07290 & 0.09710 & 0.36910 & 0.0310^{*} \\ \text { H111 } & 0.18920 & 0.37880 & 0.11450 & 0.0610^{*} \\ \text { H112 } & -0.00280 & 0.36250 & 0.06760 & 0.0610^{*} \\ \text { H113 } & 0.05800 & 0.26940 & 0.10880 & 0.0610^{*} \\ \text { H121 } & -0.21770 & 0.41140 & 0.27530 & 0.0530^{*} \\ \text { H122 } & -0.18320 & 0.28910 & 0.20350 & 0.0530^{*} \\ \text { H123 } & -0.24380 & 0.38300 & 0.16330 & 0.0530^{*}\end{array}$

Atomic displacement parameters $\left(\AA^{2}\right)$

\begin{tabular}{lllllll}
\hline & $U^{11}$ & $U^{22}$ & $U^{33}$ & $U^{12}$ & $U^{13}$ & $U^{23}$ \\
\hline Er1 & $0.0131(2)$ & $0.0219(2)$ & $0.0184(2)$ & $0.0064(2)$ & $0.0031(2)$ & $0.0079(2)$ \\
C14A & $0.092(3)$ & $0.105(3)$ & $0.061(2)$ & $0.055(2)$ & $0.052(2)$ & $0.063(2)$ \\
C14B & $0.0548(18)$ & $0.0484(17)$ & $0.0474(18)$ & $0.0099(14)$ & $-0.0065(14)$ & $-0.0183(14)$ \\
C14C & $0.0525(17)$ & $0.0285(13)$ & $0.0578(18)$ & $-0.0038(12)$ & $0.0126(13)$ & $0.0231(12)$ \\
S1 & $0.0227(11)$ & $0.0357(13)$ & $0.0211(11)$ & $0.0059(9)$ & $0.0017(8)$ & $0.0105(9)$ \\
O11 & $0.031(3)$ & $0.031(3)$ & $0.026(4)$ & $0.016(3)$ & $0.002(3)$ & $0.009(3)$ \\
O11A & $0.017(3)$ & $0.035(4)$ & $0.025(3)$ & $0.010(3)$ & $0.005(2)$ & $0.012(3)$ \\
O11B & $0.016(3)$ & $0.033(3)$ & $0.028(3)$ & $0.005(3)$ & $0.004(2)$ & $0.012(3)$ \\
O11C & $0.016(3)$ & $0.035(4)$ & $0.028(4)$ & $0.000(3)$ & $0.005(3)$ & $0.012(3)$ \\
O12A & $0.021(3)$ & $0.035(3)$ & $0.028(3)$ & $0.008(3)$ & $0.006(3)$ & $0.018(3)$ \\
O12B & $0.020(3)$ & $0.026(3)$ & $0.027(3)$ & $0.010(3)$ & $0.008(2)$ & $0.009(3)$
\end{tabular}




\begin{tabular}{|c|c|c|c|c|c|c|}
\hline $\mathrm{O} 12 \mathrm{C}$ & $0.015(3)$ & $0.017(3)$ & $0.034(4)$ & 0.005 (2) & $0.001(2)$ & 0.007 (3) \\
\hline O31A & $0.048(6)$ & 0.135 (10) & $0.064(7)$ & $0.021(6)$ & $-0.010(5)$ & 0.057 (7) \\
\hline O31B & 0.017 (4) & 0.158 (13) & $0.111(10)$ & $-0.001(6)$ & $0.005(5)$ & $-0.052(9)$ \\
\hline $\mathrm{O} 31 \mathrm{C}$ & $0.094(9)$ & 0.168 (13) & $0.041(6)$ & $-0.061(9)$ & $-0.002(6)$ & $0.035(7)$ \\
\hline $\mathrm{O} 32 \mathrm{~A}$ & $0.106(8)$ & $0.081(7)$ & $0.034(5)$ & $0.026(6)$ & $-0.011(5)$ & $0.025(5)$ \\
\hline $\mathrm{O} 32 \mathrm{~B}$ & $0.039(6)$ & $0.176(15)$ & 0.154 (14) & $0.001(8)$ & $-0.016(7)$ & $-0.092(12)$ \\
\hline $\mathrm{O} 32 \mathrm{C}$ & $0.051(6)$ & $0.119(9)$ & $0.081(7)$ & $0.017(6)$ & $0.039(5)$ & $0.064(7)$ \\
\hline N3A & $0.060(7)$ & $0.058(6)$ & $0.033(6)$ & $0.020(5)$ & $-0.009(5)$ & $0.022(5)$ \\
\hline N3B & $0.032(5)$ & $0.055(6)$ & $0.046(6)$ & $0.008(5)$ & $-0.006(4)$ & $0.004(5)$ \\
\hline N3C & $0.059(6)$ & $0.035(5)$ & $0.034(5)$ & -0.001 & $0.015(5)$ & $0.014(4)$ \\
\hline $\mathrm{C} 1 \mathrm{~A}$ & $0.022(4)$ & $0.027(4)$ & $0.020(4)$ & $0.005(4)$ & $0.005(3)$ & $0.010(4)$ \\
\hline $\mathrm{C} 1 \mathrm{~B}$ & 0.017 (4) & 0.027 (4) & $0.023(5)$ & $0.005(3)$ & $0.002(3)$ & 0.007 (4) \\
\hline $\mathrm{C} 1 \mathrm{C}$ & $0.017(4)$ & $0.026(4)$ & $0.025(5)$ & $0.004(3)$ & $0.005(3)$ & $0.010(4)$ \\
\hline $\mathrm{C} 2 \mathrm{~A}$ & $0.021(4)$ & $0.032(5)$ & $0.020(4)$ & $0.006(4)$ & $0.002(3)$ & 0.009 (4) \\
\hline $\mathrm{C} 2 \mathrm{~B}$ & $0.022(5)$ & $0.033(5)$ & $0.027(5)$ & 0.008 (4) & $0.000(4)$ & 0.007 (4) \\
\hline $\mathrm{C} 2 \mathrm{C}$ & 0.015 (4) & $0.028(5)$ & $0.030(5)$ & $0.001(3)$ & $0.003(3)$ & $0.015(4)$ \\
\hline $\mathrm{C} 3 \mathrm{~A}$ & $0.044(6)$ & $0.036(5)$ & $0.028(5)$ & $0.012(5)$ & 0.000 (4) & $0.014(4)$ \\
\hline $\mathrm{C} 3 \mathrm{~B}$ & $0.022(5)$ & $0.032(5)$ & $0.035(6)$ & $-0.003(4)$ & $0.001(4)$ & 0.007 (4) \\
\hline $\mathrm{C} 3 \mathrm{C}$ & $0.021(5)$ & $0.047(6)$ & $0.031(5)$ & $0.013(4)$ & $0.010(4)$ & $0.020(5)$ \\
\hline $\mathrm{C} 4 \mathrm{~A}$ & $0.050(6)$ & 0.047 (6) & $0.030(5)$ & $0.018(5)$ & $0.021(5)$ & $0.023(5)$ \\
\hline C4B & $0.038(6)$ & $0.034(5)$ & $0.030(5)$ & $0.003(4)$ & $0.006(4)$ & 0.007 (4) \\
\hline $\mathrm{C} 4 \mathrm{C}$ & $0.025(5)$ & $0.023(5)$ & $0.042(6)$ & $-0.004(4)$ & $0.001(4)$ & $0.022(4)$ \\
\hline $\mathrm{C} 5 \mathrm{~A}$ & $0.031(5)$ & $0.050(6)$ & $0.046(7)$ & $0.014(5)$ & $0.020(5)$ & $0.027(5)$ \\
\hline C5B & $0.033(5)$ & $0.034(5)$ & $0.036(6)$ & $0.013(4)$ & $0.010(4)$ & 0.004 (4) \\
\hline $\mathrm{C} 5 \mathrm{C}$ & $0.041(6)$ & $0.020(4)$ & $0.041(6)$ & 0.008 (4) & $0.005(5)$ & $0.014(4)$ \\
\hline C6A & $0.015(4)$ & $0.045(6)$ & $0.042(6)$ & 0.007 (4) & 0.007 (4) & $0.018(5)$ \\
\hline C6B & $0.027(5)$ & $0.031(5)$ & $0.026(5)$ & $0.012(4)$ & 0.009 (4) & 0.008 (4) \\
\hline $\mathrm{C} 6 \mathrm{C}$ & $0.029(5)$ & 0.019 (4) & $0.031(5)$ & $0.002(4)$ & $0.010(4)$ & $0.011(4)$ \\
\hline $\mathrm{C} 11$ & $0.028(5)$ & $0.069(8)$ & $0.023(5)$ & $0.014(5)$ & 0.007 (4) & $0.014(5)$ \\
\hline $\mathrm{C} 11 \mathrm{~A}$ & $0.016(4)$ & $0.024(4)$ & $0.016(4)$ & $0.001(3)$ & 0.005 & 0.009 (3) \\
\hline C11B & $0.010(4)$ & $0.021(4)$ & $0.027(5)$ & $0.007(3)$ & $0.004(3)$ & $0.012(3)$ \\
\hline $\mathrm{C} 11 \mathrm{C}$ & 0.008 (4) & $0.029(5)$ & $0.028(5)$ & $0.006(3)$ & $-0.002(3)$ & $0.016(4)$ \\
\hline C12 & $0.021(5)$ & $0.048(6)$ & $0.034(6)$ & $0.002(4)$ & $0.006(4)$ & $0.014(5)$ \\
\hline
\end{tabular}

Geometric parameters (A, $\left.{ }^{o}\right)$

\begin{tabular}{llll}
\hline Er1-O11 & $2.306(7)$ & $\mathrm{C} 1 \mathrm{~B}-\mathrm{C} 6 \mathrm{~B}$ & $1.419(14)$ \\
Er1-O11C & $2.312(8)$ & $\mathrm{C} 1 \mathrm{~B}-\mathrm{C} 11 \mathrm{~B}$ & $1.496(13)$ \\
Er1-O12A & $2.317(7)$ & $\mathrm{C} 1 \mathrm{C}-\mathrm{C} 2 \mathrm{C}$ & $1.398(13)$ \\
Er1-O12B & $2.239(6)$ & $\mathrm{C} 1 \mathrm{C}-\mathrm{C} 6 \mathrm{C}$ & $1.387(14)$ \\
Er1-O12C & $2.287(6)$ & $\mathrm{C} 1 \mathrm{C}-\mathrm{C} 11 \mathrm{C}$ & $1.507(14)$ \\
Er1-O11A & $\mathrm{C} 2 \mathrm{~A}-\mathrm{C} 3 \mathrm{~A}$ & $1.386(14)$ \\
Er1-O11B & $2.300(6)$ & $\mathrm{C} 2 \mathrm{~B}-\mathrm{C} 3 \mathrm{~B}$ & $1.390(15)$ \\
$\mathrm{C} 14 \mathrm{~A}-\mathrm{C} 4 \mathrm{~A}$ & $2.348(6)$ & $\mathrm{C} 2 \mathrm{C}-\mathrm{C} 3 \mathrm{C}$ & $1.354(16)$ \\
$\mathrm{C} 14 \mathrm{~B}-\mathrm{C} 4 \mathrm{~B}$ & $1.729(13)$ & $\mathrm{C} 3 \mathrm{~A}-\mathrm{C} 4 \mathrm{~A}$ & $1.361(17)$ \\
$\mathrm{C} 14 \mathrm{C}-\mathrm{C} 4 \mathrm{C}$ & $1.714(11)$ & $\mathrm{C} 3 \mathrm{~B}-\mathrm{C} 4 \mathrm{~B}$ & $1.383(15)$ \\
$\mathrm{S} 1-\mathrm{O} 11$ & $1.730(11)$ & $\mathrm{C} 3 \mathrm{C}-\mathrm{C} 4 \mathrm{C}$ & $1.391(15)$ \\
$\mathrm{S} 1-\mathrm{C} 11$ & $1.514(8)$ & $\mathrm{C} 4 \mathrm{~A}-\mathrm{C} 5 \mathrm{~A}$ & $1.396(16)$
\end{tabular}




\begin{tabular}{|c|c|c|c|}
\hline $\mathrm{S} 1-\mathrm{C} 12$ & $1.772(11)$ & $\mathrm{C} 4 \mathrm{~B}-\mathrm{C} 5 \mathrm{~B}$ & $1.391(15)$ \\
\hline $\mathrm{O} 11 \mathrm{~A}-\mathrm{C} 11 \mathrm{~A}$ & $1.274(11)$ & $\mathrm{C} 4 \mathrm{C}-\mathrm{C} 5 \mathrm{C}$ & $1.367(15)$ \\
\hline $\mathrm{O} 11 \mathrm{~B}-\mathrm{C} 11 \mathrm{~B}$ & $1.255(10)$ & $\mathrm{C} 5 \mathrm{~A}-\mathrm{C} 6 \mathrm{~A}$ & $1.368(16)$ \\
\hline $\mathrm{O} 11 \mathrm{C}-\mathrm{C} 11 \mathrm{C}$ & $1.255(11)$ & $\mathrm{C} 5 \mathrm{~B}-\mathrm{C} 6 \mathrm{~B}$ & $1.394(15)$ \\
\hline $\mathrm{O} 12 \mathrm{~A}-\mathrm{C} 11 \mathrm{~A}$ & $1.250(10)$ & $\mathrm{C} 5 \mathrm{C}-\mathrm{C} 6 \mathrm{C}$ & $1.391(15)$ \\
\hline $\mathrm{O} 12 \mathrm{~B}-\mathrm{C} 11 \mathrm{~B}$ & $1.249(10)$ & $\mathrm{C} 2 \mathrm{~A}-\mathrm{H} 2 \mathrm{~A}$ & 0.9500 \\
\hline $\mathrm{O} 12 \mathrm{C}-\mathrm{C} 11 \mathrm{C}$ & $1.271(12)$ & $\mathrm{C} 2 \mathrm{~B}-\mathrm{H} 2 \mathrm{~B}$ & 0.9500 \\
\hline O31A-N3A & $1.206(17)$ & $\mathrm{C} 2 \mathrm{C}-\mathrm{H} 2 \mathrm{C}$ & 0.9500 \\
\hline $\mathrm{O} 31 \mathrm{~B}-\mathrm{N} 3 \mathrm{~B}$ & $1.151(16)$ & $\mathrm{C} 5 \mathrm{~A}-\mathrm{H} 5 \mathrm{~A}$ & 0.9500 \\
\hline $\mathrm{O} 31 \mathrm{C}-\mathrm{N} 3 \mathrm{C}$ & $1.191(16)$ & $\mathrm{C} 5 \mathrm{~B}-\mathrm{H} 5 \mathrm{~B}$ & 0.9500 \\
\hline $\mathrm{O} 32 \mathrm{~A}-\mathrm{N} 3 \mathrm{~A}$ & $1.229(14)$ & $\mathrm{C} 5 \mathrm{C}-\mathrm{H} 5 \mathrm{C}$ & 0.9500 \\
\hline $\mathrm{O} 32 \mathrm{~B}-\mathrm{N} 3 \mathrm{~B}$ & $1.13(2)$ & $\mathrm{C} 6 \mathrm{~A}-\mathrm{H} 6 \mathrm{~A}$ & 0.9500 \\
\hline $\mathrm{O} 32 \mathrm{C}-\mathrm{N} 3 \mathrm{C}$ & $1.188(15)$ & $\mathrm{C} 6 \mathrm{~B}-\mathrm{H} 6 \mathrm{~B}$ & 0.9500 \\
\hline $\mathrm{N} 3 \mathrm{~A}-\mathrm{C} 3 \mathrm{~A}$ & $1.480(16)$ & $\mathrm{C} 6 \mathrm{C}-\mathrm{H} 6 \mathrm{C}$ & 0.9500 \\
\hline $\mathrm{N} 3 \mathrm{~B}-\mathrm{C} 3 \mathrm{~B}$ & $1.474(14)$ & $\mathrm{C} 11-\mathrm{H} 111$ & 0.9800 \\
\hline $\mathrm{N} 3 \mathrm{C}-\mathrm{C} 3 \mathrm{C}$ & $1.481(14)$ & $\mathrm{C} 11-\mathrm{H} 112$ & 0.9800 \\
\hline $\mathrm{C} 1 \mathrm{~A}-\mathrm{C} 2 \mathrm{~A}$ & $1.380(13)$ & $\mathrm{C} 11-\mathrm{H} 113$ & 0.9800 \\
\hline $\mathrm{C} 1 \mathrm{~A}-\mathrm{C} 6 \mathrm{~A}$ & $1.386(14)$ & $\mathrm{C} 12-\mathrm{H} 121$ & 0.9800 \\
\hline $\mathrm{C} 1 \mathrm{~A}-\mathrm{C} 11 \mathrm{~A}$ & $1.524(13)$ & $\mathrm{C} 12-\mathrm{H} 122$ & 0.9800 \\
\hline $\mathrm{C} 1 \mathrm{~B}-\mathrm{C} 2 \mathrm{~B}$ & $1.369(13)$ & $\mathrm{C} 12-\mathrm{H} 123$ & 0.9800 \\
\hline $\mathrm{O} 11-\mathrm{Er} 1-\mathrm{O} 11 \mathrm{C}$ & $72.5(3)$ & $\mathrm{N} 3 \mathrm{C}-\mathrm{C} 3 \mathrm{C}-\mathrm{C} 2 \mathrm{C}$ & $117.7(9)$ \\
\hline $\mathrm{O} 11-\mathrm{Er} 1-\mathrm{O} 12 \mathrm{~A}$ & $74.7(2)$ & $\mathrm{N} 3 \mathrm{C}-\mathrm{C} 3 \mathrm{C}-\mathrm{C} 4 \mathrm{C}$ & $120.7(10)$ \\
\hline $\mathrm{O} 11-\mathrm{Er} 1-\mathrm{O} 12 \mathrm{~B}$ & $80.6(2)$ & $\mathrm{C} 2 \mathrm{C}-\mathrm{C} 3 \mathrm{C}-\mathrm{C} 4 \mathrm{C}$ & $121.5(9)$ \\
\hline $\mathrm{O} 11-\mathrm{Er} 1-\mathrm{O} 12 \mathrm{C}^{\mathrm{i}}$ & $77.0(3)$ & $\mathrm{C} 14 \mathrm{~A}-\mathrm{C} 4 \mathrm{~A}-\mathrm{C} 3 \mathrm{~A}$ & $124.2(9)$ \\
\hline $\mathrm{O} 11-\mathrm{Er} 1-\mathrm{O} 11 \mathrm{~A}^{\mathrm{ii}}$ & $140.9(3)$ & $\mathrm{C} 14 \mathrm{~A}-\mathrm{C} 4 \mathrm{~A}-\mathrm{C} 5 \mathrm{~A}$ & $117.3(9)$ \\
\hline $\mathrm{O} 11-\mathrm{Er} 1-\mathrm{O} 11 \mathrm{~B}^{\mathrm{ii}}$ & $143.3(2)$ & $\mathrm{C} 3 \mathrm{~A}-\mathrm{C} 4 \mathrm{~A}-\mathrm{C} 5 \mathrm{~A}$ & $118.5(11)$ \\
\hline $\mathrm{O} 11 \mathrm{C}-\mathrm{Er} 1-\mathrm{O} 12 \mathrm{~A}$ & $145.4(2)$ & $\mathrm{C} 14 \mathrm{~B}-\mathrm{C} 4 \mathrm{~B}-\mathrm{C} 3 \mathrm{~B}$ & $124.4(8)$ \\
\hline $\mathrm{O} 11 \mathrm{C}-\mathrm{E} 1-\mathrm{O} 12 \mathrm{~B}$ & $84.1(2)$ & $\mathrm{Cl} 4 \mathrm{~B}-\mathrm{C} 4 \mathrm{~B}-\mathrm{C} 5 \mathrm{~B}$ & $116.3(8)$ \\
\hline $\mathrm{O} 11 \mathrm{C}-\mathrm{Er} 1-\mathrm{O} 12 \mathrm{C}^{\mathrm{i}}$ & $94.7(2)$ & $\mathrm{C} 3 \mathrm{~B}-\mathrm{C} 4 \mathrm{~B}-\mathrm{C} 5 \mathrm{~B}$ & $119.3(10)$ \\
\hline $\mathrm{O} 11 \mathrm{~A}^{\mathrm{ii}}-\mathrm{Er} 1-\mathrm{O} 11 \mathrm{C}$ & $73.9(2)$ & $\mathrm{C} 14 \mathrm{C}-\mathrm{C} 4 \mathrm{C}-\mathrm{C} 3 \mathrm{C}$ & $121.1(8)$ \\
\hline $\mathrm{O} 11 \mathrm{~B}^{\mathrm{ii}}-\mathrm{E} 1-\mathrm{O} 11 \mathrm{C}$ & $130.3(2)$ & $\mathrm{C} 14 \mathrm{C}-\mathrm{C} 4 \mathrm{C}-\mathrm{C} 5 \mathrm{C}$ & $119.7(9)$ \\
\hline $\mathrm{O} 12 \mathrm{~A}-\mathrm{E} 1-\mathrm{O} 12 \mathrm{~B}$ & $80.0(2)$ & $\mathrm{C} 3 \mathrm{C}-\mathrm{C} 4 \mathrm{C}-\mathrm{C} 5 \mathrm{C}$ & $119.2(10)$ \\
\hline $\mathrm{O} 12 \mathrm{~A}-\mathrm{E} 1-\mathrm{O} 12 \mathrm{C}^{\mathrm{i}}$ & $88.4(2)$ & $\mathrm{C} 4 \mathrm{~A}-\mathrm{C} 5 \mathrm{~A}-\mathrm{C} 6 \mathrm{~A}$ & $121.0(10)$ \\
\hline $\mathrm{O} 11 \mathrm{~A}^{\mathrm{ii}}-\mathrm{Er} 1-\mathrm{O} 12 \mathrm{~A}$ & $130.5(2)$ & $\mathrm{C} 4 \mathrm{~B}-\mathrm{C} 5 \mathrm{~B}-\mathrm{C} 6 \mathrm{~B}$ & $120.5(10)$ \\
\hline $\mathrm{O} 11 \mathrm{~B}^{\mathrm{ii}}-\mathrm{Er} 1-\mathrm{O} 12 \mathrm{~A}$ & $83.5(2)$ & $\mathrm{C} 4 \mathrm{C}-\mathrm{C} 5 \mathrm{C}-\mathrm{C} 6 \mathrm{C}$ & $120.1(10)$ \\
\hline $\mathrm{O} 12 \mathrm{~B}-\mathrm{Er} 1-\mathrm{O} 12 \mathrm{C}^{\mathrm{i}}$ & $156.8(2)$ & $\mathrm{C} 1 \mathrm{~A}-\mathrm{C} 6 \mathrm{~A}-\mathrm{C} 5 \mathrm{~A}$ & $119.9(9)$ \\
\hline $\mathrm{O} 11 \mathrm{~A}^{\mathrm{ii}}-\mathrm{E} 1-\mathrm{O} 12 \mathrm{~B}$ & $76.7(2)$ & $\mathrm{C} 1 \mathrm{~B}-\mathrm{C} 6 \mathrm{~B}-\mathrm{C} 5 \mathrm{~B}$ & $119.3(9)$ \\
\hline $\mathrm{O} 11 \mathrm{~B}^{\mathrm{ii}-}-\mathrm{E} 1-\mathrm{O} 12 \mathrm{~B}$ & $124.6(2)$ & $\mathrm{C} 1 \mathrm{C}-\mathrm{C} 6 \mathrm{C}-\mathrm{C} 5 \mathrm{C}$ & $120.3(9)$ \\
\hline $\mathrm{O} 11 \mathrm{~A}^{\mathrm{ii}}-\mathrm{Er} 1-\mathrm{O} 12 \mathrm{C}^{\mathrm{i}}$ & $125.3(2)$ & $\mathrm{O} 11 \mathrm{~A}-\mathrm{C} 11 \mathrm{~A}-\mathrm{O} 12 \mathrm{~A}$ & $127.7(8)$ \\
\hline $\mathrm{O} 11 \mathrm{~B}^{\mathrm{ii}}-\mathrm{E} 1-\mathrm{O} 12 \mathrm{C}^{\mathrm{i}}$ & $73.2(2)$ & $\mathrm{O} 11 \mathrm{~A}-\mathrm{C} 11 \mathrm{~A}-\mathrm{C} 1 \mathrm{~A}$ & $116.0(7)$ \\
\hline $\mathrm{O} 11 \mathrm{~A}^{\mathrm{ii}}-\mathrm{E} 1-\mathrm{O} 11 \mathrm{~B}^{\mathrm{ii}}$ & $75.2(2)$ & $\mathrm{O} 12 \mathrm{~A}-\mathrm{C} 11 \mathrm{~A}-\mathrm{C} 1 \mathrm{~A}$ & $116.3(8)$ \\
\hline $\mathrm{O} 11-\mathrm{S} 1-\mathrm{C} 11$ & $103.9(5)$ & $\mathrm{O} 11 \mathrm{~B}-\mathrm{C} 11 \mathrm{~B}-\mathrm{O} 12 \mathrm{~B}$ & $121.6(8)$ \\
\hline $\mathrm{O} 11-\mathrm{S} 1-\mathrm{C} 12$ & $106.0(5)$ & $\mathrm{O} 11 \mathrm{~B}-\mathrm{C} 11 \mathrm{~B}-\mathrm{C} 1 \mathrm{~B}$ & $119.8(8)$ \\
\hline $\mathrm{C} 11-\mathrm{S} 1-\mathrm{C} 12$ & $99.3(5)$ & $\mathrm{O} 12 \mathrm{~B}-\mathrm{C} 11 \mathrm{~B}-\mathrm{C} 1 \mathrm{~B}$ & $118.6(8)$ \\
\hline $\mathrm{Er} 1-\mathrm{O} 11-\mathrm{S} 1$ & $133.1(4)$ & $\mathrm{O} 11 \mathrm{C}-\mathrm{C} 11 \mathrm{C}-\mathrm{O} 12 \mathrm{C}$ & $123.6(9)$ \\
\hline $\mathrm{Er} 1 \mathrm{ii}-\mathrm{O} 11 \mathrm{~A}-\mathrm{C} 11 \mathrm{~A}$ & $140.3(6)$ & $\mathrm{O} 11 \mathrm{C}-\mathrm{C} 11 \mathrm{C}-\mathrm{C} 1 \mathrm{C}$ & $118.1(8)$ \\
\hline $\mathrm{Er} 1 \mathrm{ii}-\mathrm{O} 11 \mathrm{~B}-\mathrm{C} 11 \mathrm{~B}$ & $110.9(5)$ & $\mathrm{O} 12 \mathrm{C}-\mathrm{C} 11 \mathrm{C}-\mathrm{C} 1 \mathrm{C}$ & $118.3(8)$ \\
\hline
\end{tabular}




\begin{tabular}{|c|c|}
\hline $\mathrm{Er} 1-\mathrm{O} 11 \mathrm{C}-\mathrm{C} 11 \mathrm{C}$ & $113.9(6)$ \\
\hline $\mathrm{Er} 1-\mathrm{O} 12 \mathrm{~A}-\mathrm{C} 11 \mathrm{~A}$ & $132.8(6)$ \\
\hline $\mathrm{Er} 1-\mathrm{O} 12 \mathrm{~B}-\mathrm{C} 11 \mathrm{~B}$ & $172.3(6)$ \\
\hline $\mathrm{Er} 1 \mathrm{i}-\mathrm{O} 12 \mathrm{C}-\mathrm{C} 11 \mathrm{C}$ & $128.2(6)$ \\
\hline $\mathrm{O} 31 \mathrm{~A}-\mathrm{N} 3 \mathrm{~A}-\mathrm{O} 32 \mathrm{~A}$ & $124.3(12)$ \\
\hline $\mathrm{O} 31 \mathrm{~A}-\mathrm{N} 3 \mathrm{~A}-\mathrm{C} 3 \mathrm{~A}$ & $118.5(10)$ \\
\hline $\mathrm{O} 32 \mathrm{~A}-\mathrm{N} 3 \mathrm{~A}-\mathrm{C} 3 \mathrm{~A}$ & $117.1(12)$ \\
\hline $\mathrm{O} 31 \mathrm{~B}-\mathrm{N} 3 \mathrm{~B}-\mathrm{O} 32 \mathrm{~B}$ & $118.3(13)$ \\
\hline $\mathrm{O} 31 \mathrm{~B}-\mathrm{N} 3 \mathrm{~B}-\mathrm{C} 3 \mathrm{~B}$ & $120.3(12)$ \\
\hline $\mathrm{O} 32 \mathrm{~B}-\mathrm{N} 3 \mathrm{~B}-\mathrm{C} 3 \mathrm{~B}$ & $121.2(11)$ \\
\hline $\mathrm{O} 31 \mathrm{C}-\mathrm{N} 3 \mathrm{C}-\mathrm{O} 32 \mathrm{C}$ & $124.1(12)$ \\
\hline $\mathrm{O} 31 \mathrm{C}-\mathrm{N} 3 \mathrm{C}-\mathrm{C} 3 \mathrm{C}$ & $116.7(11)$ \\
\hline $\mathrm{O} 32 \mathrm{C}-\mathrm{N} 3 \mathrm{C}-\mathrm{C} 3 \mathrm{C}$ & $119.2(10)$ \\
\hline $\mathrm{C} 2 \mathrm{~A}-\mathrm{C} 1 \mathrm{~A}-\mathrm{C} 6 \mathrm{~A}$ & $119.6(9)$ \\
\hline $\mathrm{C} 2 \mathrm{~A}-\mathrm{C} 1 \mathrm{~A}-\mathrm{C} 11 \mathrm{~A}$ & $120.3(8)$ \\
\hline $\mathrm{C} 6 \mathrm{~A}-\mathrm{C} 1 \mathrm{~A}-\mathrm{C} 11 \mathrm{~A}$ & $120.1(8)$ \\
\hline $\mathrm{C} 2 \mathrm{~B}-\mathrm{C} 1 \mathrm{~B}-\mathrm{C} 6 \mathrm{~B}$ & $119.5(9)$ \\
\hline $\mathrm{C} 2 \mathrm{~B}-\mathrm{C} 1 \mathrm{~B}-\mathrm{C} 11 \mathrm{~B}$ & $121.6(8)$ \\
\hline $\mathrm{C} 6 \mathrm{~B}-\mathrm{C} 1 \mathrm{~B}-\mathrm{C} 11 \mathrm{~B}$ & $118.9(8)$ \\
\hline $\mathrm{C} 2 \mathrm{C}-\mathrm{C} 1 \mathrm{C}-\mathrm{C} 6 \mathrm{C}$ & $119.0(9)$ \\
\hline $\mathrm{C} 2 \mathrm{C}-\mathrm{C} 1 \mathrm{C}-\mathrm{C} 11 \mathrm{C}$ & $120.7(8)$ \\
\hline $\mathrm{C} 6 \mathrm{C}-\mathrm{C} 1 \mathrm{C}-\mathrm{C} 11 \mathrm{C}$ & $120.3(8)$ \\
\hline $\mathrm{C} 1 \mathrm{~A}-\mathrm{C} 2 \mathrm{~A}-\mathrm{C} 3 \mathrm{~A}$ & $119.6(9)$ \\
\hline $\mathrm{C} 1 \mathrm{~B}-\mathrm{C} 2 \mathrm{~B}-\mathrm{C} 3 \mathrm{~B}$ & $120.6(9)$ \\
\hline $\mathrm{C} 1 \mathrm{C}-\mathrm{C} 2 \mathrm{C}-\mathrm{C} 3 \mathrm{C}$ & $119.8(9)$ \\
\hline $\mathrm{N} 3 \mathrm{~A}-\mathrm{C} 3 \mathrm{~A}-\mathrm{C} 2 \mathrm{~A}$ & $115.0(10)$ \\
\hline $\mathrm{N} 3 \mathrm{~A}-\mathrm{C} 3 \mathrm{~A}-\mathrm{C} 4 \mathrm{~A}$ & $123.6(10)$ \\
\hline $\mathrm{C} 2 \mathrm{~A}-\mathrm{C} 3 \mathrm{~A}-\mathrm{C} 4 \mathrm{~A}$ & $121.4(10)$ \\
\hline $\mathrm{N} 3 \mathrm{~B}-\mathrm{C} 3 \mathrm{~B}-\mathrm{C} 2 \mathrm{~B}$ & $116.5(9)$ \\
\hline $\mathrm{N} 3 \mathrm{~B}-\mathrm{C} 3 \mathrm{~B}-\mathrm{C} 4 \mathrm{~B}$ & $122.7(10)$ \\
\hline $\mathrm{C} 2 \mathrm{~B}-\mathrm{C} 3 \mathrm{~B}-\mathrm{C} 4 \mathrm{~B}$ & $120.7(9)$ \\
\hline $\mathrm{O} 11 \mathrm{C}-\mathrm{Er} 1-\mathrm{O} 11-\mathrm{S} 1$ & $123.8(6)$ \\
\hline $\mathrm{O} 12 \mathrm{~A}-\mathrm{Er} 1-\mathrm{O} 11-\mathrm{S} 1$ & $-67.4(6)$ \\
\hline $\mathrm{O} 12 \mathrm{~B}-\mathrm{Er} 1-\mathrm{O} 11-\mathrm{S} 1$ & $-149.5(6)$ \\
\hline $\mathrm{O} 12 \mathrm{C}-\mathrm{Er} 1-\mathrm{O} 11-\mathrm{S} 1$ & $24.6(5)$ \\
\hline 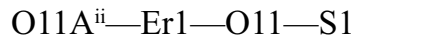 & $155.6(4)$ \\
\hline $\mathrm{O} 11 \mathrm{~B}^{\mathrm{ii}}-\mathrm{E} 1-\mathrm{O} 11-\mathrm{S} 1$ & $-11.7(8)$ \\
\hline $\mathrm{O} 11-\mathrm{Er} 1-\mathrm{O} 11 \mathrm{C}-\mathrm{C} 11 \mathrm{C}$ & $-136.0(7)$ \\
\hline $\mathrm{O} 12 \mathrm{~A}-\mathrm{Er} 1-\mathrm{O} 11 \mathrm{C}-\mathrm{C} 11 \mathrm{C}$ & $-155.2(6)$ \\
\hline $\mathrm{O} 12 \mathrm{~B}-\mathrm{Er} 1-\mathrm{O} 11 \mathrm{C}-\mathrm{C} 11 \mathrm{C}$ & $142.1(6)$ \\
\hline $\mathrm{O} 12 \mathrm{C}-\mathrm{E} 1-\mathrm{O} 11 \mathrm{C}-\mathrm{C} 11 \mathrm{C}$ & $-61.2(6)$ \\
\hline $\mathrm{O} 11 \mathrm{~A}-\mathrm{ii}-\mathrm{E} 1-\mathrm{O} 11 \mathrm{C}-\mathrm{C} 11 \mathrm{C}$ & $64.2(6)$ \\
\hline $\mathrm{O} 11 \mathrm{~B}^{\mathrm{ii}}-\mathrm{Er} 1-\mathrm{O} 11 \mathrm{C}-\mathrm{C} 11 \mathrm{C}$ & $10.6(7)$ \\
\hline $\mathrm{O} 11-\mathrm{Er} 1-\mathrm{O} 12 \mathrm{~A}-\mathrm{C} 11 \mathrm{~A}$ & $-102.6(8)$ \\
\hline $\mathrm{O} 11 \mathrm{C}-\mathrm{Er} 1-\mathrm{O} 12 \mathrm{~A}-\mathrm{C} 11 \mathrm{~A}$ & $-83.6(8)$ \\
\hline $\mathrm{O} 12 \mathrm{~B}-\mathrm{Er} 1-\mathrm{O} 12 \mathrm{~A}-\mathrm{C} 11 \mathrm{~A}$ & $-19.8(7)$ \\
\hline $\mathrm{O} 12 \mathrm{C}-\mathrm{Er} 1-\mathrm{O} 12 \mathrm{~A}-\mathrm{C} 11 \mathrm{~A}$ & $-179.6(8)$ \\
\hline
\end{tabular}

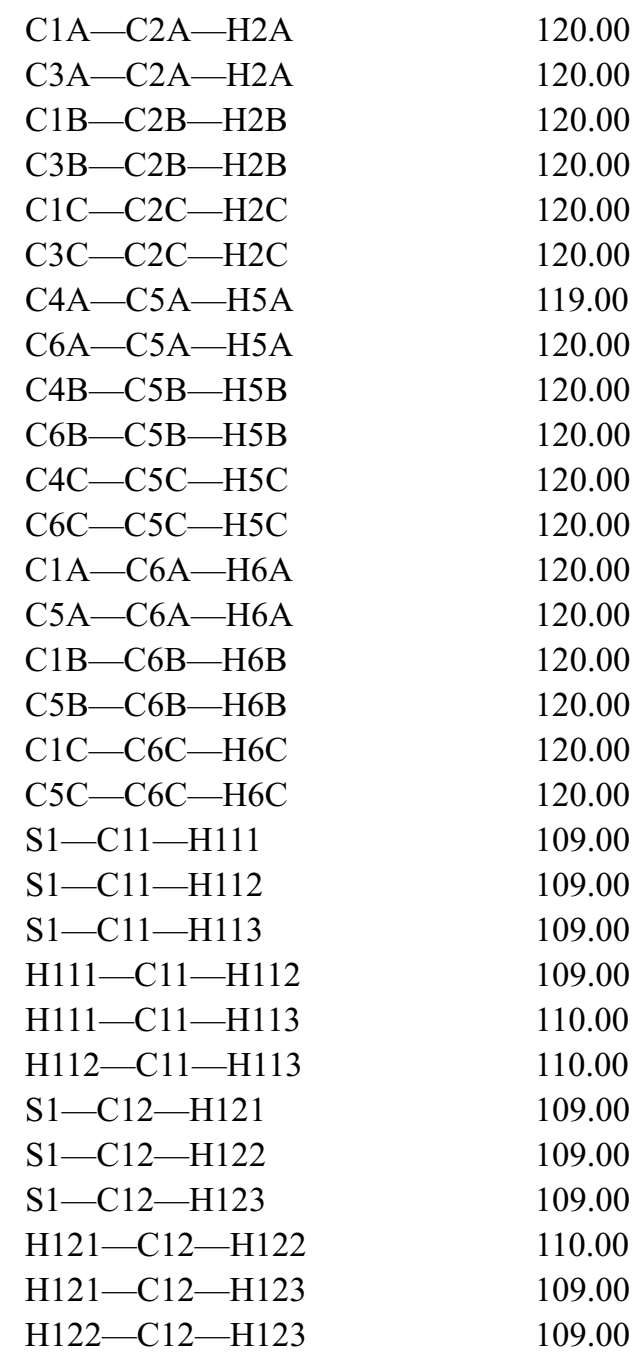




\begin{tabular}{|c|c|}
\hline $\mathrm{O} 11 \mathrm{~A}-\mathrm{Er} 1-\mathrm{O} 12 \mathrm{~A}-\mathrm{C} 11 \mathrm{~A}$ & $43.0(8)$ \\
\hline $\mathrm{O} 11 \mathrm{~B}^{\mathrm{ii}}-\mathrm{Er} 1-\mathrm{O} 12 \mathrm{~A}-\mathrm{C} 11 \mathrm{~A}$ & $107.2(8)$ \\
\hline $\mathrm{O} 11-\mathrm{E} 1-\mathrm{O} 12 \mathrm{C}^{\mathrm{i}}-\mathrm{C} 11 \mathrm{C}^{\mathrm{i}}$ & $-162.8(8)$ \\
\hline $\mathrm{O} 11 \mathrm{C}-\mathrm{Er} 1-\mathrm{O} 12 \mathrm{C}^{\mathrm{i}}-\mathrm{C} 11 \mathrm{C}^{\mathrm{i}}$ & $126.4(8)$ \\
\hline $\mathrm{O} 12 \mathrm{~A}-\mathrm{Er} 1-\mathrm{O} 12 \mathrm{C}-\mathrm{C} 11 \mathrm{C}^{\mathrm{i}}$ & $-88.1(8)$ \\
\hline $\mathrm{O} 12 \mathrm{~B}-\mathrm{E} 1-\mathrm{O} 12 \mathrm{C}^{\mathrm{i}}-\mathrm{C} 11 \mathrm{C}^{\mathrm{i}}$ & $-147.7(7)$ \\
\hline $\mathrm{O} 11-\mathrm{Er} 1-\mathrm{O} 11 \mathrm{~A}^{\mathrm{ii}}-\mathrm{C}_{11 \mathrm{~A}^{\mathrm{ii}}}$ & $85.7(10)$ \\
\hline $\mathrm{O} 11 \mathrm{C}-\mathrm{Er} 1-\mathrm{O} 11 \mathrm{~A}-\mathrm{ii}-\mathrm{C}^{\mathrm{ii}}$ & $117.2(10)$ \\
\hline $\mathrm{O} 12 \mathrm{~A}-\mathrm{Er} 1-\mathrm{O} 11 \mathrm{~A}-\mathrm{C} 11 \mathrm{~A}^{\mathrm{ii}}$ & $-34.5(11)$ \\
\hline $\mathrm{O} 12 \mathrm{~B}-\mathrm{Er} 1-\mathrm{O} 11 \mathrm{~A}^{\mathrm{ii}}-\mathrm{C} 11 \mathrm{~A}^{\mathrm{ii}}$ & $29.6(9)$ \\
\hline $\mathrm{O} 11-\mathrm{Er} 1-\mathrm{O} 11 \mathrm{~B}^{\mathrm{ii}}-\mathrm{C} 11 \mathrm{~B}^{\mathrm{ii}}$ & $-118.1(6)$ \\
\hline $\mathrm{O} 11 \mathrm{C}-\mathrm{Er} 1-\mathrm{O} 11 \mathrm{~B}^{\mathrm{ii}}-\mathrm{C} 11 \mathrm{~B}^{\mathrm{ii}}$ & $123.2(6)$ \\
\hline $\mathrm{O} 12 \mathrm{~A}-\mathrm{Er} 1-\mathrm{O} 11 \mathrm{~B}^{\mathrm{ii}}-\mathrm{C} 11 \mathrm{~B}^{\mathrm{ii}}$ & $-64.8(6)$ \\
\hline $\mathrm{O} 12 \mathrm{~B}-\mathrm{Er} 1-\mathrm{O} 11 \mathrm{~B}^{\mathrm{ii}}-\mathrm{C} 11 \mathrm{~B}^{\mathrm{ii}}$ & $8.3(7)$ \\
\hline $\mathrm{C} 11-\mathrm{S} 1-\mathrm{O} 11-\mathrm{Er} 1$ & $154.8(6)$ \\
\hline $\mathrm{C} 12-\mathrm{S} 1-\mathrm{O} 11-\mathrm{Er} 1$ & $-101.1(6)$ \\
\hline $\mathrm{Er} 1{ }^{\mathrm{ii}}-\mathrm{O} 11 \mathrm{~A}-\mathrm{C} 11 \mathrm{~A}-\mathrm{O} 12 \mathrm{~A}$ & $-5.6(16)$ \\
\hline $\mathrm{Er} 1{ }^{\mathrm{ii}}-\mathrm{O} 11 \mathrm{~A}-\mathrm{C} 11 \mathrm{~A}-\mathrm{C} 1 \mathrm{~A}$ & $175.9(7)$ \\
\hline $\mathrm{Er} \mathrm{i}^{\mathrm{i}}-\mathrm{O} 11 \mathrm{~B}-\mathrm{C} 11 \mathrm{~B}-\mathrm{O} 12 \mathrm{~B}$ & $-0.5(11)$ \\
\hline 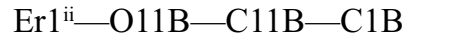 & $178.3(7)$ \\
\hline $\mathrm{Er} 1-\mathrm{O} 11 \mathrm{C}-\mathrm{C} 11 \mathrm{C}-\mathrm{O} 12 \mathrm{C}$ & $14.1(11)$ \\
\hline $\mathrm{Er} 1-\mathrm{O} 11 \mathrm{C}-\mathrm{C} 11 \mathrm{C}-\mathrm{C} 1 \mathrm{C}$ & $-164.6(6)$ \\
\hline $\mathrm{Er} 1-\mathrm{O} 12 \mathrm{~A}-\mathrm{C} 11 \mathrm{~A}-\mathrm{O} 11 \mathrm{~A}$ & $-27.7(14)$ \\
\hline $\mathrm{Er} 1-\mathrm{O} 12 \mathrm{~A}-\mathrm{C} 11 \mathrm{~A}-\mathrm{C} 1 \mathrm{~A}$ & $150.8(6)$ \\
\hline $\mathrm{Er} 1 \mathrm{i}-\mathrm{O} 12 \mathrm{C}-\mathrm{C} 11 \mathrm{C}-\mathrm{O} 11 \mathrm{C}$ & $95.6(9)$ \\
\hline $\mathrm{Er} 1 \mathrm{i}-\mathrm{O} 12 \mathrm{C}-\mathrm{C} 11 \mathrm{C}-\mathrm{C} 1 \mathrm{C}$ & $-85.7(9)$ \\
\hline $\mathrm{O} 32 \mathrm{~A}-\mathrm{N} 3 \mathrm{~A}-\mathrm{C} 3 \mathrm{~A}-\mathrm{C} 2 \mathrm{~A}$ & $-150.4(12)$ \\
\hline $\mathrm{O} 31 \mathrm{~A}-\mathrm{N} 3 \mathrm{~A}-\mathrm{C} 3 \mathrm{~A}-\mathrm{C} 4 \mathrm{~A}$ & $-154.6(14)$ \\
\hline $\mathrm{O} 32 \mathrm{~A}-\mathrm{N} 3 \mathrm{~A}-\mathrm{C} 3 \mathrm{~A}-\mathrm{C} 4 \mathrm{~A}$ & $27.9(18)$ \\
\hline $\mathrm{O} 31 \mathrm{~A}-\mathrm{N} 3 \mathrm{~A}-\mathrm{C} 3 \mathrm{~A}-\mathrm{C} 2 \mathrm{~A}$ & $27.2(17)$ \\
\hline $\mathrm{O} 32 \mathrm{~B}-\mathrm{N} 3 \mathrm{~B}-\mathrm{C} 3 \mathrm{~B}-\mathrm{C} 4 \mathrm{~B}$ & $-5(2)$ \\
\hline $\mathrm{O} 31 \mathrm{~B}-\mathrm{N} 3 \mathrm{~B}-\mathrm{C} 3 \mathrm{~B}-\mathrm{C} 2 \mathrm{~B}$ & $-0.8(19)$ \\
\hline $\mathrm{O} 31 \mathrm{~B}-\mathrm{N} 3 \mathrm{~B}-\mathrm{C} 3 \mathrm{~B}-\mathrm{C} 4 \mathrm{~B}$ & $-179.9(14)$ \\
\hline $\mathrm{O} 32 \mathrm{~B}-\mathrm{N} 3 \mathrm{~B}-\mathrm{C} 3 \mathrm{~B}-\mathrm{C} 2 \mathrm{~B}$ & $174.1(16)$ \\
\hline $\mathrm{O} 32 \mathrm{C}-\mathrm{N} 3 \mathrm{C}-\mathrm{C} 3 \mathrm{C}-\mathrm{C} 4 \mathrm{C}$ & $-62.2(16)$ \\
\hline $\mathrm{O} 31 \mathrm{C}-\mathrm{N} 3 \mathrm{C}-\mathrm{C} 3 \mathrm{C}-\mathrm{C} 4 \mathrm{C}$ & $119.4(14)$ \\
\hline $\mathrm{O} 32 \mathrm{C}-\mathrm{N} 3 \mathrm{C}-\mathrm{C} 3 \mathrm{C}-\mathrm{C} 2 \mathrm{C}$ & $120.3(13)$ \\
\hline
\end{tabular}

$\begin{array}{ll}\mathrm{C} 6 \mathrm{~B}-\mathrm{C} 1 \mathrm{~B}-\mathrm{C} 11 \mathrm{~B}-\mathrm{O} 12 \mathrm{~B} & 174.7(9) \\ \mathrm{C} 6 \mathrm{C}-\mathrm{C} 1 \mathrm{C}-\mathrm{C} 11 \mathrm{C}-\mathrm{O} 11 \mathrm{C} & -18.6(13) \\ \mathrm{C} 2 \mathrm{C}-\mathrm{C} 1 \mathrm{C}-\mathrm{C} 11 \mathrm{C}-\mathrm{O} 11 \mathrm{C} & 160.3(8) \\ \mathrm{C} 2 \mathrm{C}-\mathrm{C} 1 \mathrm{C}-\mathrm{C} 11 \mathrm{C}-\mathrm{O} 12 \mathrm{C} & -18.5(12) \\ \mathrm{C} 11 \mathrm{C}-\mathrm{C} 1 \mathrm{C}-\mathrm{C} 2 \mathrm{C}-\mathrm{C} 3 \mathrm{C} & -179.3(9) \\ \mathrm{C} 6 \mathrm{C}-\mathrm{C} 1 \mathrm{C}-\mathrm{C} 11 \mathrm{C}-\mathrm{O} 12 \mathrm{C} & 162.7(9) \\ \mathrm{C} 2 \mathrm{C}-\mathrm{C} 1 \mathrm{C}-\mathrm{C} 6 \mathrm{C}-\mathrm{C} 5 \mathrm{C} & 1.5(14) \\ \mathrm{C} 6 \mathrm{C}-\mathrm{C} 1 \mathrm{C}-\mathrm{C} 2 \mathrm{C}-\mathrm{C} 3 \mathrm{C} & -0.5(14) \\ \mathrm{C} 11 \mathrm{C}-\mathrm{C} 1 \mathrm{C}-\mathrm{C} 6 \mathrm{C}-\mathrm{C} 5 \mathrm{C} & -179.6(9) \\ \mathrm{C} 1 \mathrm{~A}-\mathrm{C} 2 \mathrm{~A}-\mathrm{C} 3 \mathrm{~A}-\mathrm{C} 4 \mathrm{~A} & -5.3(17) \\ \mathrm{C} 1 \mathrm{~A}-\mathrm{C} 2 \mathrm{~A}-\mathrm{C} 3 \mathrm{~A}-\mathrm{N} 3 \mathrm{~A} & 173.0(10) \\ \mathrm{C} 1 \mathrm{~B}-\mathrm{C} 2 \mathrm{~B}-\mathrm{C} 3 \mathrm{~B}-\mathrm{C} 4 \mathrm{~B} & -3.0(17) \\ \mathrm{C} 1 \mathrm{~B}-\mathrm{C} 2 \mathrm{~B}-\mathrm{C} 3 \mathrm{~B}-\mathrm{N} 3 \mathrm{~B} & 177.8(10) \\ \mathrm{C} 1 \mathrm{C}-\mathrm{C} 2 \mathrm{C}-\mathrm{C} 3 \mathrm{C}-\mathrm{N} 3 \mathrm{C} & 177.0(9) \\ \mathrm{C} 1 \mathrm{C}-\mathrm{C} 2 \mathrm{C}-\mathrm{C} 3 \mathrm{C}-\mathrm{C} 4 \mathrm{C} & -0.4(15) \\ \mathrm{C} 2 \mathrm{~A}-\mathrm{C} 3 \mathrm{~A}-\mathrm{C} 4 \mathrm{~A}-\mathrm{C} 14 \mathrm{~A} & -174.7(9) \\ \mathrm{C} 2 \mathrm{~A}-\mathrm{C} 3 \mathrm{~A}-\mathrm{C} 4 \mathrm{~A}-\mathrm{C} 5 \mathrm{~A} & 2.9(18) \\ \mathrm{N} 3 \mathrm{~A}-\mathrm{C} 3 \mathrm{~A}-\mathrm{C} 4 \mathrm{~A}-\mathrm{C} 5 \mathrm{~A} & -175.2(11) \\ \mathrm{N} 3 \mathrm{~A}-\mathrm{C} 3 \mathrm{~A}-\mathrm{C} 4 \mathrm{~A}-\mathrm{C} 14 \mathrm{~A} & 7.2(18) \\ \mathrm{N} 3 \mathrm{~B}-\mathrm{C} 3 \mathrm{~B}-\mathrm{C} 4 \mathrm{~B}-\mathrm{C} 5 \mathrm{~B} & -177.1(11) \\ \mathrm{C} 2 \mathrm{~B}-\mathrm{C} 3 \mathrm{~B}-\mathrm{C} 4 \mathrm{~B}-\mathrm{C} 14 \mathrm{~B} & -178.6(9) \\ \mathrm{C} 2 \mathrm{~B}-\mathrm{C} 3 \mathrm{~B}-\mathrm{C} 4 \mathrm{~B}-\mathrm{C} 5 \mathrm{~B} & 3.8(17) \\ \mathrm{N} 3 \mathrm{~B}-\mathrm{C} 3 \mathrm{~B}-\mathrm{C} 4 \mathrm{~B}-\mathrm{C} 14 \mathrm{~B} & 0.6(17) \\ \mathrm{N} 3 \mathrm{C}-\mathrm{C} 3 \mathrm{C}-\mathrm{C} 4 \mathrm{C}-\mathrm{C} 14 \mathrm{C} & 1.6(14) \\ \mathrm{N} 3 \mathrm{C}-\mathrm{C} 3 \mathrm{C}-\mathrm{C} 4 \mathrm{C}-\mathrm{C} 5 \mathrm{C} & -177.1(10) \\ \mathrm{C} 2 \mathrm{C}-\mathrm{C} 3 \mathrm{C}-\mathrm{C} 4 \mathrm{C}-\mathrm{C} 14 \mathrm{C} & 178.9(8) \\ \mathrm{C} 2 \mathrm{C}-\mathrm{C} 3 \mathrm{C}-\mathrm{C} 4 \mathrm{C}-\mathrm{C} 5 \mathrm{C} & 0.2(15) \\ \mathrm{C} 14 \mathrm{~A}-\mathrm{C} 4 \mathrm{~A}-\mathrm{C} 5 \mathrm{~A}-\mathrm{C} 6 \mathrm{~A} & 178.2(10) \\ \mathrm{C} 3 \mathrm{~A}-\mathrm{C} 4 \mathrm{~A}-\mathrm{C} 5 \mathrm{~A}-\mathrm{C} 6 \mathrm{~A} & 0.5(19) \\ \mathrm{C} 14 \mathrm{~B}-\mathrm{C} 4 \mathrm{~B}-\mathrm{C} 5 \mathrm{~B}-\mathrm{C} 6 \mathrm{~B} & -179.9(9) \\ \mathrm{C} 3 \mathrm{~B}-\mathrm{C} 4 \mathrm{~B}-\mathrm{C} 5 \mathrm{~B}-\mathrm{C} 6 \mathrm{~B} & -2.0(17) \\ \mathrm{C} 14 \mathrm{C}-\mathrm{C} 4 \mathrm{C}-\mathrm{C} 5 \mathrm{C}-\mathrm{C} 6 \mathrm{C} & -177.9(8) \\ \mathrm{C} 3 \mathrm{C}-\mathrm{C} 4 \mathrm{C}-\mathrm{C} 5 \mathrm{C}-\mathrm{C} 6 \mathrm{C} & 0.9(16) \\ \mathrm{C} 4 \mathrm{~A}-\mathrm{C} 5 \mathrm{~A}-\mathrm{C} 6 \mathrm{~A}-\mathrm{C} 1 \mathrm{~A} & -1.5(18) \\ \mathrm{C} 4 \mathrm{~B}-\mathrm{C} 5 \mathrm{~B}-\mathrm{C} 6 \mathrm{~B}-\mathrm{C} 1 \mathrm{~B} & -0.5(16) \\ \mathrm{C} 4 \mathrm{C}-\mathrm{C} 5 \mathrm{C}-\mathrm{C} 6 \mathrm{C}-\mathrm{C} 1 \mathrm{C} & -1.7(16) \\ & \end{array}$

Symmetry codes: (i) $-x,-y+1,-z+1$; (ii) $-x+1,-y+1,-z+1$.

Hydrogen-bond geometry $\left(\AA,{ }^{\circ}\right)$

\begin{tabular}{lllll}
\hline$D-\mathrm{H} \cdots A$ & $D-\mathrm{H}$ & $\mathrm{H} \cdots A$ & $D \cdots A$ & $D-\mathrm{H} \cdots A$ \\
\hline $\mathrm{C} 2 A-\mathrm{H} 2 A \cdots \mathrm{S} 1$ & 0.95 & 2.86 & $3.743(10)$ & 155 \\
$\mathrm{C} 2 B-\mathrm{H} 2 B \cdots \mathrm{O} 11$ & 0.95 & 2.56 & $3.298(13)$ & 135 \\
$\mathrm{C} 11-\mathrm{H} 111 \cdots \mathrm{C} 14 A^{\mathrm{iii}}$ & 0.98 & 2.79 & $3.486(11)$ & 129 \\
$\mathrm{C} 12-\mathrm{H} 123 \cdots \mathrm{O} 22 A^{\text {iv }}$ & 0.98 & 2.44 & $3.376(15)$ & 158 \\
\hline
\end{tabular}

Symmetry codes: (iii) $-x+1,-y+1,-z$; (iv) $-x,-y+1,-z$. 